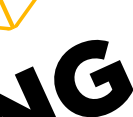 \\ 西
}

\section{work}

\section{COVID 19 and Mental Health Deterioration among BAME groups in the UK}

\author{
Eugenio Proto
}

Climent Quintana-Domeque

\section{Working Paper}

2020-054

$07 / 2020$

HUMAN CAPITAL AND

ECONOMIC OPPORTUNITY

GLOBAL WORKING GROUP
The University of Chicago 1126 E. 59th Street Box 107 Chicago IL 60637 


\title{
COVID-19 and Mental Health Deterioration among BAME groups in the UK ${ }^{*}$
}

\author{
Eugenio Proto, University of Glasgow, CEPR and IZA \\ Climent Quintana-Domeque, University of Exeter, HCEO and IZA
}

First draft: 16 July 2020

\begin{abstract}
We use the UK Household Longitudinal Study and compare pre- (2017-2019) and post-COVID-19 data (April 2020) for the same group of individuals to assess and quantify changes in mental health among ethnic groups in the UK. We confirm the previously documented average deterioration in mental health for the whole sample of individuals interviewed pre- and post-COVID-19, and uncover four new facts. First, ethnicity predicts mental health deterioration when interacted with gender. Among men, BAME individuals experience a higher deterioration in mental health compared to British White individuals. However, among women, the deterioration in mental health is similar for both BAME and British White individuals. Second, the gender gap in mental health deterioration is only present among British White individuals and not among BAME individuals. Third, the drop in mental health among women and BAME men is very similar. Finally, there is substantial heterogeneity across BAME groups. The BAME group of Bangladeshi, Indian and Pakistani appears to be driving the difference in the gender gap in mental health deterioration between British White and BAME individuals. We call for additional research on the effects of the COVID-19 pandemic across different ethnic groups, and urge both policy makers and researchers to allocate resources to collect larger sample sizes of minority ethnic groups.
\end{abstract}

Keywords: GHQ-12, wellbeing, mental health, mental distress, ethnicity, gender. JEL codes: I1, J1, J15.

\footnotetext{
* If you have comments, questions or suggestions, contact Eugenio Proto (eugenio.proto@glasgow.ac.uk) and/or Climent Quintana-Domeque (c.quintana-domeque@exeter.ac.uk).
} 


\section{Introduction}

The COVID-19 pandemic and the associated lockdown measures are having heterogeneous effects across individuals in the population. One outcome for which differential impacts have been documented in the UK is psychological wellbeing. This is an interesting outcome for economists and policymakers alike, if only because of the well-established link between productivity and psychological wellbeing (see Proto, 2016, for a review). While a lot has already been written about the differential effects of the COVID-19 pandemic by age and gender (Banks and $\mathrm{Xu}, 2020$; Davillas and Jones, 2020; Etheridge and Spantig, 2020; Oreffice and Quintana-Domeque, 2020), much less is known about how its effects vary across ethnics groups (Daly et al., 2020). In this paper we ask whether BAME (Black, Asian, and minority ethnic) individuals have suffered from a stronger deterioration in mental health compared to British White individuals.

Using the UK Household Longitudinal Study (UKHLS) and comparing preand post-COVID-19 data (April 2020) for the same group of individuals, Banks and $\mathrm{Xu}$ (2020) find a much larger reduction in women's mental health relative to men's as measured by the 12-item General Health Questionnaire (GHQ-12). ${ }^{1}$ With regards to age, Banks and Xu (2020) and Daly et al. (2020) have uncovered larger drops in mental health among younger individuals. The focus in this paper is neither age nor gender but ethnicity. We are motivated by the "growing concerns that the UK's minority ethnic groups are being disproportionately affected" by the COVID-19 pandemic (e.g., Kirby, 2020; Public Health England, 2020; Razaq et al. 2020), "mainly through exposure to infection and health risks (including mortality) and through exposure to loss of income" (Platt and Warwick, 2020).

To the best of our knowledge Daly et al. (2020) is the only study focusing on ethnic differences in mental health changes pre- and post-COVID-19 in the UK. Adjusting for age, sex, marital status, education, income and vulnerability to the

\footnotetext{
1 This stronger deterioration of mental health among women is also documented by Daly et al. (2020) and Etheridge and Spanting (2020), both of them using the UKHLS. Davillas and Jones (2020) use also the UKHLS and show that age and gender are the main explanatory factors behind GHQ-12 inequality. Oreffice and Quintana-Domeque (2020) document gender differences across socioeconomic and wellbeing dimensions, including mental health, after three months of lockdown (June 2020) in the UK using an online sample of approximately 1,500 respondents.
} 
health effects of COVID-19, they report no differential changes in mental health problems between White and non-White individuals from 2017-2019 to April $2020 .^{2}$ The authors also note that "it will be important for future research to identify the mental health burden associated with COVID-19 in BAME groups", and in this paper we follow their steps.

Using the UKHLS, we investigate differential changes in mental health from 2017-2019 to April 2020 between British White individuals and individuals in BAME groups, and among British White, Other White, Mixed, Black, BIP (Bangladeshi, Indian and Pakistani), Chinese and other Asian, and Arab individuals. We also study whether there are differential changes by gender within ethnic groups.

We find that BAME individuals suffered an increase in mental distress of 0.26 SD, while British White individuals suffered an increase of 0.21 SD. However, the $0.05-\mathrm{SD}$ differential increase is not statistically significant [95\% CI: -0.005 , 0.097]. This finding is consistent with the lack of an ethnic gap in mental health deterioration when comparing White vs. non-White in Daly et al. (2020). However, once we allow ethnicity to interact with gender, a more complex picture emerges, and we uncover four new facts.

First, ethnicity predicts mental health deterioration when interacted with gender: among males, BAME individuals suffer a higher deterioration in mental health than British White individuals; however, among females, the deterioration in mental health is similar for both BAME and British White individuals; (ii) a direct consequence of the first fact is that the gender gap in mental health deterioration is only present among British White individuals and not among BAME individuals; (iii) the drop in mental health among women and BAME men is very similar; (iv) there is substantial heterogeneity across BAME groups: the BAME group of Bangladeshi, Indian and Pakistani appears to be driving the difference in the gender gap in mental health deterioration between British White and BAME individuals.

\footnotetext{
2 They actually find that White's mental health problems (i.e., having a GHQ-12 "caseness" score $\geq 3$ ) experienced a non-statistically significant increase of 3 percentage points [95\% CI: $(-1.7,7.1)$, p-value $=0.234]$ above that of non-Whites.
} 
While physical health and mortality has been the focus of previous reports and studies on the differential effects of COVID-19 by ethnicity (e.g., Public Health England, 2020a), our study highlights the importance of focusing on another key dimension of health and wellbeing: mental health.

Our analysis reveals other interesting patterns. In our sample of the general population, we find that people living in London and Scotland have suffered a worse deterioration in mental health (0.8 and 0.9 standard deviations more) compared to individuals living in England (excluding London), and that selfemployed and retired individuals have been affected (0.8 and 0.9 standard deviations) more than employed individuals; however, within our sample of working individuals, the geographical patterns disappear once we adjust for type of job, and find that those classified as "Small Employers or Own Account" have suffered a larger drop in mental health ( $\sim 0.20$ standard deviations) compared to individuals classified as working in "Management and Professional" occupations. Neither marital status nor household size predicts changes in mental health. Similarly, both net personal income and being classified as clinically vulnerable to the health effects of COVID-19 are irrelevant predictors of mental health deterioration.

Reassuringly, our findings are consistent with the previous studies using the UKHLS (e.g., Banks and $\mathrm{Xu}, 2020$ ). We show that the 14,289 individuals in the UKHLS who were interviewed both in 2017-2019 and in April 2020 experienced an increase in mental distress of 0.22 standard deviations (95\% CI: [0.20, 0.23]) as measured by the GHQ-12 (0-36): from 11.14 in 2017-2019 to 12.38 in April 2020. We also confirm the findings on the gender gap in the deterioration of mental health (e.g., Etheridge and Spantig, 2020): women suffered a higher increase (0.27 SD) in mental distress compared to men (0.14), a differential increase of $0.13 \mathrm{SD}$ (95\% CI: $[0.10,0.16])$.

Perhaps one of the main limitations of our preliminary study is the relatively small size of the subsample of BAME participants $(\mathrm{N}=2,015)$ in comparison to the full sample $(\mathrm{N}=14,155)$. However, this caveat also highlights what we believe to be an important take away of this first investigation: we need much larger samples of minority ethnic groups in the UKHLS so that properly- 
powered statistical analyses can be carried out. We call for additional research on the potential differential effects of the COVID-19 pandemic by ethnicity, and urge both policy makers and researchers to allocate resources to collect larger sample sizes of minority ethnic groups. One way forward to start gathering this type of data is via online surveys launched on platforms such as Prolific, which allow researchers to select participants based on existing characteristics such as ethnicity. Ensuring that large samples of different ethnic groups are available is a necessary first step to conduct well-powered statistical investigations.

Yet another possibility is to combine all the monthly COVID waves when they are available. Of course, such an approach is not without its perils. While statistical power will be gained at the time of conducting pre- and post-COVID-19 comparisons, the scope for analysing potential dynamic aspects will be lost.

Section 2 describes the dataset, the sample and the variables used in our analysis. Section 3 contains the regression analysis showing the differences in changes in mental health from 2017-2019 to April 2020 among ethnic groups after adjusting for socioeconomic and sociodemographic characteristics. Section 4 concludes. 


\section{Data description}

We use two waves of data from the UK Household Longitudinal Study (UKHLS or Understanding Society), wave 9 (2017-2019) and the first monthly COVID-19 wave (April 2020) and join others (Banks and Xu, 2020; Daly et al., 2020; Davillas and Jones, 2020; Etheridge and Spantig, 2020) in their effort to understand the effects of the COVID-19 pandemic and the lockdown on mental wellbeing. We do not use sampling weights or make any adjustment for survey design other than clustering standard errors at the individual level when required. ${ }^{3}$

\subsection{The 12-item General Health Questionnaire}

In order to measure the effects on mental wellbeing we use the 12-item General Health Questionnaire, GHQ-12 (Goldberg and Williams, 1988). The GHQ-12 is a well-known self-report instrument for evaluating mental health where the respondent must report the extent to which 12 symptoms are present in the past few weeks on a Likert scale (see Appendix A for additional information), and its range goes from 0 to 36 . We standardise the within-individual change in GHQ-12 between waves, so that differences in GHQ-12 among ethnic groups are measured in standard deviations.

Following Daly et al. (2020), we also use the GHQ-12 to dichotomise whether respondents are at risk of presenting with mental health problems. If individuals report experiencing at least 3 of the 12 symptoms, we classify them at risk of mental health problems (see Appendix A for additional information). This approach is used in Daly et al. (2020), who note that "the cut-off threshold has been validated against psychiatric interviews for the detection of psychological disorders" (Aalato et al., 2012; Goldberg et al., 1997).

\footnotetext{
${ }^{3}$ More details about the UKHLS and its sampling methodology can be found in Daly et al. (2020) and the references therein.
} 


\subsection{Sample}

We combine the two waves to generate a dataset of 53,507 observations with two components, one cross-sectional, the other longitudinal, and drop 4,624 observations with missing information on the GHQ-12. Table 1 reports how many observations belong to each wave and how many individuals are observed in both waves, revealing that $43 \%$ of the individuals interviewed in 2017-2019 $(14,289 / 33,143)$ were re-interviewed in April 2020. Reassuringly, Daly et al. (2020) report that mental health problems in 2017-2019 are not related to participation in the COVID-19 wave.

Table 1: Cross-sectional and longitudinal dimensions

Individual is observed twice

\begin{tabular}{lccc}
\cline { 2 - 4 } Wave & No & Yes & Total \\
\hline 2017-2019 & 18,854 & 14,289 & 33,143 \\
April 2020 & 1,451 & 14,289 & 15,740 \\
& & & \\
\hline Total & 20,305 & 28,578 & 48,883 \\
\hline
\end{tabular}

\subsection{Descriptive statistics}

Pooling the two cross-sections of data (Observations $=48,883 ; \mathrm{N}$ (individuals) $=34,594$ ), we find that mental distress (GHQ-12: 0-36) has increased from 11.32 in Wave 9 (2017-2019) to 12.48 in April 2020, a 0.20 standard deviation increase [95\% CI: 0.19, 0.22].4 Exploiting the longitudinal dimension of the dataset (i.e., comparing the same individual before and after: observations $=28,578 ; \mathrm{N}=$ $14,289)$, we document a similar change, from 11.14 to 12.38 , a 0.22 standard deviation increase [95\% CI: $0.20,0.23] .{ }^{5}$ In what follows we focus on the longitudinal dimension of the dataset.

\footnotetext{
${ }^{4}$ Standard errors clustered at the individual level.

${ }^{5}$ Standard errors clustered at the individual level.
} 
Table 2 contains the summary statistics of our sample of (panel) individuals (information on the construction of the variables is provided in the Appendix). The average GHQ-12 at baseline is 11.14 and 22\% of individuals are likely to have mental health problems as judged by the GHQ 'caseness' indicator. 7.5\% of individuals are younger than 25, 22\% of individuals are 65 or older, and $58 \%$ of individuals are females.

With regards to ethnicity, $86 \%$ of our sample respondents are British White (British, English, Scottish, Welsh, Northern Ireland), about 5\% are Other White (Irish, Gypsy or Irish traveller, any other white background), 1.5\% are Mixed (White and Black Caribbean, White and Black African, White and Asian, any other mixed background), $1.8 \%$ are Black (Caribbean, African, any other black background), 5\% are BIP (Indian, Pakistani, Bangladeshi), 1\% are Chinese or any other Asian background, and $0.2 \%$ are Arab.

While all participants in the April 2020 wave were interviewed online, Wave 9 was conducted in three different modes: $53.87 \%$ online, $45.47 \%$ face-toface and $0.67 \%$ phone. In the table we can see that $28 \%$ of the panel individuals were interviewed face-to-face at baseline.

In terms of household structure and marital status, we can see that the average household size is 1.9 and that $72 \%$ of the respondents live with a partner (married or not). With regards to place of residence, $71 \%$ of respondents live in England (excluding London), 9.7\% in London, 8.9\% in Scotland, 5.8\% in Wales and $4.3 \%$ in Northern Ireland.

Moving to education and employment, the table shows that $36 \%$ of the respondents have at least a BA degree, 8.3\% were self-employed at baseline, $54.9 \%$ were employed at baseline, and $23.8 \%$ were retired. In terms of income and health status, the average total net personal (monthly) income is $£ 1,792$ and $37.3 \%$ of individuals belong to the COVID-19 high-risk group (i.e., individuals who are considered clinically vulnerable to the health effects of COVID-19).

Finally, among individuals who were either employed or self-employed at baseline, 50\% work in management \& professional occupations, $21 \%$ work in semi-routine and routine jobs, and 15\% work in intermediate type of jobs. 
Table 2: Descriptive statistics of variables among (panel) individuals

\begin{tabular}{|c|c|c|c|c|c|}
\hline \multirow[b]{2}{*}{ GHQ-12 (at baseline) } & & & & & \\
\hline & 14,289 & 11.143 & 5.381 & 1 & 36 \\
\hline GHQ 'caseness' score (at baseline) & 14,289 & 0.220 & 0.414 & 0 & 1 \\
\hline$<25$ & 14,289 & 0.075 & 0.263 & 0 & 1 \\
\hline $25-34$ & 14,289 & 0.113 & 0.316 & 0 & 1 \\
\hline $35-44$ & 14,289 & 0.162 & 0.369 & 0 & 1 \\
\hline $45-54$ & 14,289 & 0.214 & 0.410 & 0 & 1 \\
\hline $55-64$ & 14,289 & 0.218 & 0.413 & 0 & 1 \\
\hline $65+$ & 14,289 & 0.219 & 0.414 & 0 & 1 \\
\hline Female & 14,289 & 0.582 & 0.493 & 0 & 1 \\
\hline British White & 14,155 & 0.858 & 0.349 & 0 & 1 \\
\hline Other White & 14,155 & 0.047 & 0.211 & 0 & 1 \\
\hline Mixed & 14,155 & 0.015 & 0.122 & 0 & 1 \\
\hline Black & 14,155 & 0.018 & 0.134 & 0 & 1 \\
\hline BIP & 14,155 & 0.050 & 0.218 & 0 & 1 \\
\hline Chinese \& other Asian Background & 14,155 & 0.010 & 0.101 & 0 & 1 \\
\hline Arab & 14,155 & 0.002 & 0.042 & 0 & 1 \\
\hline Face-to-Face interview (at baseline) & 14,289 & 0.284 & 0.451 & 0 & 1 \\
\hline Household size (at follow-up) & 14,289 & 1.881 & 1.364 & 0 & 13 \\
\hline Living with a partner & 14,252 & 0.720 & 0.449 & 0 & 1 \\
\hline Widowed & 14,252 & 0.034 & 0.182 & 0 & 1 \\
\hline Divorced or Separated & 14,252 & 0.081 & 0.272 & 0 & 1 \\
\hline Never married & 14,252 & 0.165 & 0.372 & 0 & 1 \\
\hline England (excluding London) & 14,280 & 0.712 & 0.453 & 0 & 1 \\
\hline London & 14,280 & 0.097 & 0.297 & 0 & 1 \\
\hline Wales & 14,280 & 0.058 & 0.234 & 0 & 1 \\
\hline Scotland & 14,280 & 0.089 & 0.285 & 0 & 1 \\
\hline Northern Ireland & 14,280 & 0.043 & 0.203 & 0 & 1 \\
\hline BA or higher & 12,537 & 0.360 & 0.480 & 0 & 1 \\
\hline Diploma or equiv. & 12,537 & 0.120 & 0.325 & 0 & 1 \\
\hline A level or equiv. & 12,537 & 0.117 & 0.321 & 0 & 1 \\
\hline GCSE or equiv. & 12,537 & 0.247 & 0.431 & 0 & 1 \\
\hline None & 12,537 & 0.156 & 0.363 & 0 & 1 \\
\hline Self-employed (at baseline) & 13,874 & 0.083 & 0.277 & 0 & 1 \\
\hline Employed (at baseline) & 13,874 & 0.549 & 0.498 & 0 & 1 \\
\hline Unemployed (at baseline) & 13,874 & 0.024 & 0.154 & 0 & 1 \\
\hline Retired (at baseline) & 13,874 & 0.238 & 0.426 & 0 & 1 \\
\hline House-keeper (at baseline) & 13,874 & 0.037 & 0.189 & 0 & 1 \\
\hline Student (at baseline) & 13,874 & 0.040 & 0.196 & 0 & 1 \\
\hline Disabled (at baseline) & 13,874 & 0.022 & 0.145 & 0 & 1 \\
\hline Other(at baseline) & 13,874 & 0.007 & 0.082 & 0 & 1 \\
\hline Total net personal income - no deductions (in $£ 1,000$ ) & 14,289 & 1.792 & 1.766 & -0.625 & 65.645 \\
\hline
\end{tabular}




\begin{tabular}{lccccc}
\hline COVID-19 high-risk group & 14,288 & 0.373 & 0.484 & 0 & 1 \\
\hline Management \& professional (at follow-up) & 8,821 & 0.503 & 0.500 & 0 & 1 \\
Intermediate (at follow-up) & 8,821 & 0.147 & 0.355 & 0 & 1 \\
Small employers \& own account (at follow-up) & 8,821 & 0.087 & 0.281 & 0 & 1 \\
Lower supervisory \& technical (at follow-up) & 8,821 & 0.057 & 0.231 & 0 & 1 \\
Semi-routine \& routine (at follow-up) & 8,821 & 0.206 & 0.405 & 0 & 1 \\
\hline
\end{tabular}

Figure 1 displays the average mental distress (on a scale from 0-36) in 2017-2019 and April 2020 across demographic groups: females vs. males, BAME vs. British White individuals, females vs. males within BAME individuals, and females vs. males within British White individuals.

Figure 1: Mental distress (GHQ) in 2017-2019 and April 2020 by demographic groups
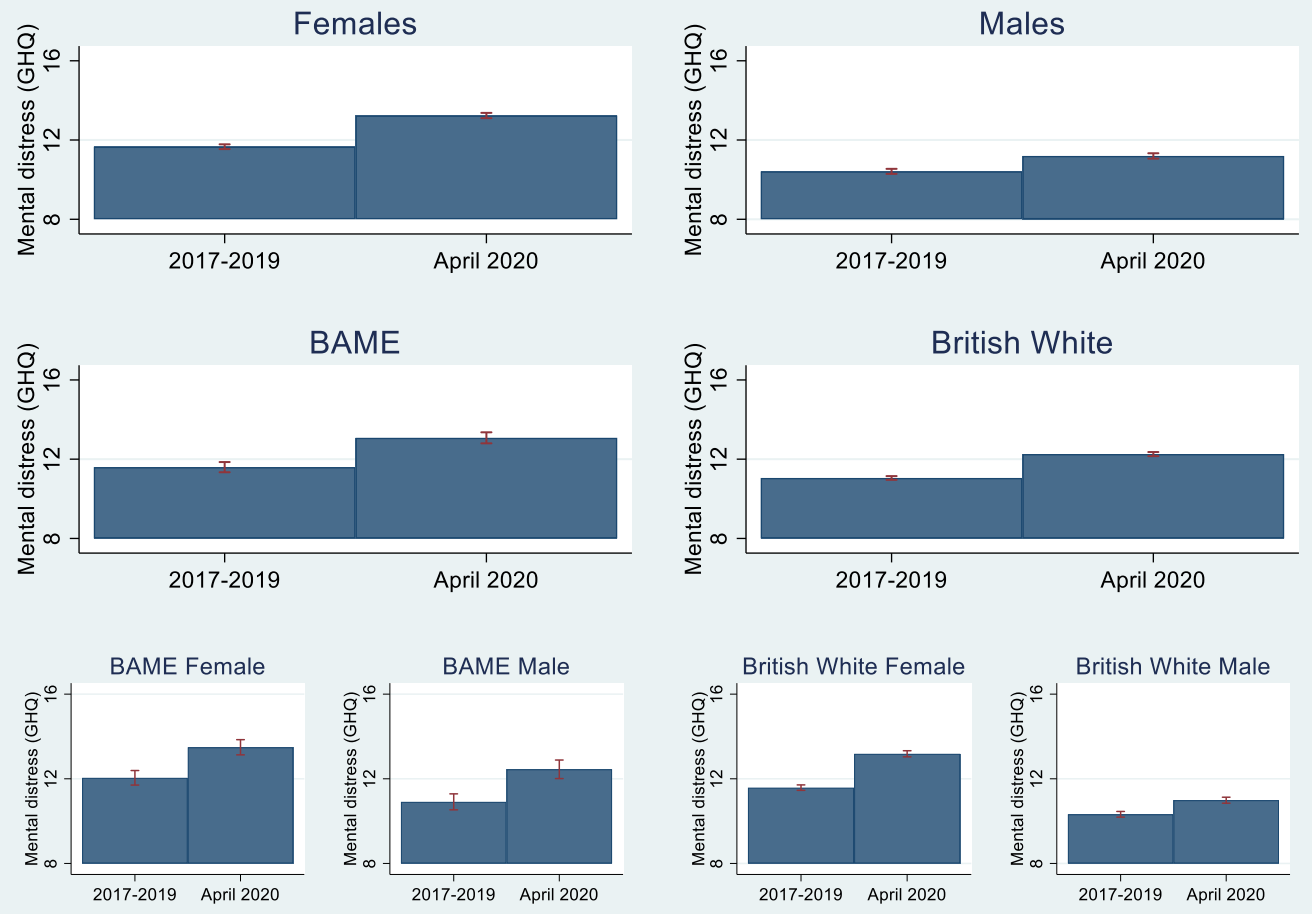

Note: $95 \%$ lower and upper confidence bounds are found as the mean of the mental distress score for the particular demographic group \pm the square root of "the standard deviation of the mental distress score/number of individuals in the demographic group (n)" times the two-sided t statistic corresponding to a significance level $\alpha=0.05$ with (n-1) degrees of freedom. 
Looking at the top panel in Figure 1 we find that women suffered a higher increase in mental distress compared to men: 0.27 standard deviations [95\% CI: $0.25,0.30]$ vs. 0.14 SD [95\% CI: $0.11,0.16$ ], a differential increase of 0.13 SD [95\% CI: $0.10,0.16]$. This finding confirms the previously documented gender gap in the deterioration of mental health (Banks and Xu, 2020; Daly et al., 2020; Davillas and Jones, 2020; Etheridge and Spantig, 2020).

The intermediate panel in Figure 1 focuses on ethnicity. The bar graphs show that BAME individuals suffered an increase in mental distress of 0.26 SD [95\% CI: 0.21, 0.31], while British White individuals suffered an increase of 0.21 SD [95\% CI: $0.19,0.23]$. The $0.05-S D$ differential increase is not statistically significant [95\% CI: -0.005, 0.097], consistent with the finding in Daly et al. (2020) when comparing White vs. non-White individuals.

Finally, the bottom panel in Figure 1 enquires about the interaction between gender and ethnicity. Perhaps the most remarkable pattern in this panel is that the gender gap in mental distress increase is only present among British White individuals (0.16 SD [95\% CI: 0.12, 0.19]) and not among BAME individuals (-0.02 SD [95\% CI: $-0.11,0.08])$.

This first exploration of the data reveals three stylised facts. First, women, regardless of their ethnicity, experienced a higher deterioration in mental health compared to British White men. Second, BAME men experienced a higher deterioration in mental health compared to British White men. Finally, the deterioration in mental health among women and BAME men is very similar. 


\section{Regression analysis}

This section contains a series of ordinary least squares (OLS) regressions to assess the relevance of potential predictors of changes in mental distress and mental health problems from 2017-2019 to April 2020, with a particular focus on ethnicity and its interaction with gender, among two different groups of individuals, the general population and the working population (i.e. employed and self-employed individuals).

We use OLS to estimate linear regressions of the following form:

$$
\Delta y_{i}=\alpha+\text { BFemale }_{i}+\gamma B A M E_{i}+\delta\left(\text { Female }_{i} \times B A M E_{i}\right)+\pi \boldsymbol{X}_{\boldsymbol{i}}+e_{i},
$$

where $\Delta y_{i} \equiv y_{\text {April2020,i }}-y_{2017-2019, i}$ is either the standardised difference in mental distress (GHQ-12) or the difference in the GHQ 'caseness' score (at least 3 symptoms out of 12) from 2017-2019 to April 2020 for individual $i$; Female $_{i}=1$ if individual $i$ is a woman, 0 if the individual is a man; $B A M E_{i}=1$ if individual $i$ belongs to the BAME group, 0 else; $\boldsymbol{X}_{\boldsymbol{i}}$ is a vector of other demographic and socioeconomic variables, including age indicators, interview fixed effects, household size, marital status indicators, place of residence indicators, qualification indicators, employment status indicators, personal income, a vulnerability to the health effects of COVID-19 indicator, and occupation indicators when focusing on working individuals; $e_{i}$ is a regression residual.

\subsection{Changes in mental distress in the general population}

\subsubsection{Differences between BAME and British White}

In Table 3 we study the predictors of standardised changes in mental distress from 2017-2019 to April 2020. In column (1) we assess whether being an individual in a BAME group predicts the change in mental distress after adjusting for age and gender, and accounting for household size and interview differences (i.e., month of interview fixed effects in 2017-2019 and whether the mode of interview in 20172019 was face-to-face). The coefficient on the BAME group indicator is small (0.025) and not statistically significant $(\mathrm{SE}=0.026)$, consistent with the findings 
in Daly et al. (2020). ${ }^{6}$ We also confirm previous findings with regards to age and gender (Banks and Xu, 2020; Davillas and Jones, 2020; Daly et al., 2020; Etheridge and Spantig, 2020): younger individuals and females have experienced larger increases in mental distress compared to older individuals and males.

Column (1) also reveals that those who were interviewed face-to-face in 2017-2019 have experienced a higher increase (0.04 SD) in mental distress compared to those who were interviewed online. Given that the April 2020 wave was conducted online for everyone, this finding is consistent with a mode of survey administration effect (Dolan and Kavetsos, 2016): individuals are more likely to report mental distress if interviewed online than face-to-face. Indeed, Dolan and Kavetsos (2016) find that individuals consistently report higher subjective wellbeing over the phone compared to face-to-face interviews using data from the April 2011 to March 2012 Annual Population Survey in the UK.

In column (2) we add the interaction between the BAME ethnicity indicator and gender, and find three interesting results. First, the coefficient on BAME experiences a dramatic jump from $0.025(\mathrm{SE}=0.026)$ to $0.133(\mathrm{SE}=0.038)$, indicating that among BAME male individuals mental distress actually increased by 0.133 SD. Second, the coefficient on female increases from $0.124(\mathrm{SE}=0.017)$ to 0.149 (SE = 0.018). Finally, the coefficient on the interaction between BAME and gender is $-0.180(\mathrm{SE}=0.051)$. Taking into account both the main "effects" and the interaction "effects", we find that: (a) among males, BAME individuals experience a higher increase $(0.133$ SD, 95\% CI: [0.059,0.207]) in mental distress compared to British White individuals; (b) among females, BAME individuals experience a nonstatistically significant lower increase (-0.047 SD, 95\% CI: [-0.116,0.023]) in mental distress compared to British White individuals; (c) among British White individuals, females experience a higher increase (0.149 SD, 95\% CI: [$0.114,0.183])$ ) in mental distress compared to men; (d) among BAME individuals, females experience a non-statistically significant lower increase (-0.031 SD, 95\% CI: $[-0.125,0.063])$ in mental distress compared to men. The bottom line of all these interactions is that (as shown in Figure 1 as well) the increase in mental

\footnotetext{
6 Table A1 in Appendix C uses an indicator for White vs. non-White individuals as Daly et al. (2020).
} 
distress among BAME individuals does not differ by gender: 0.133 SD [95\% CI: $0.059,0.207]$ among males, 0.102 SD [95\% CI: 0.032, 0.172] among females.

In columns (3), (4) and (5) we include additional demographic and socioeconomic variables: marital status indicators, place of residence indicators (England (excluding London), London, Wales, Scotland and Northern Ireland), highest-qualification indicators, employment status indicators, net personal income and a vulnerability to the health effects of COVID-19 indicator. The previous documented relationships between gender and ethnicity are robust to the inclusion of all these variables. In addition we find larger increases ( $\sim 0.09$ SD) in mental distress among (1) individuals living in London or Scotland compared to those living in England (London excluded) and among (2) individuals who are selfemployed or retired compared to those who are employed. Moreover, the increases in mental distress are much lower (-0.36 SD) among disabled individuals. Neither personal net income nor the COVID-19 high risk indicator predicts changes in mental distress.

Table 3: OLS regressions of (standardised) changes in mental distress in the UKHLS from Wave 9 (20172019) to April 2020 on BAME and other characteristics

(1)

\begin{tabular}{lccccc}
\hline & & & & \\
& $0.116^{* * *}$ & $0.117^{* * *}$ & 0.061 & 0.104 & 0.091 \\
& $(0.043)$ & $(0.043)$ & $(0.056)$ & $(0.074)$ & $(0.076)$ \\
& $0.105^{* * *}$ & $0.106^{* * *}$ & $0.071^{*}$ & $0.150^{* * *}$ & $0.138^{* * *}$ \\
$35-44$ & $(0.032)$ & $(0.032)$ & $(0.036)$ & $(0.046)$ & $(0.049)$ \\
& 0.000 & 0.000 & -0.014 & $0.073^{*}$ & 0.062 \\
$45-54$ & $(0.030)$ & $(0.030)$ & $(0.033)$ & $(0.042)$ & $(0.045)$ \\
& $-0.117^{* * *}$ & $-0.119^{* * *}$ & $-0.139^{* * *}$ & -0.053 & -0.063 \\
$55-64$ & $(0.025)$ & $(0.025)$ & $(0.028)$ & $(0.038)$ & $(0.040)$ \\
& $-0.055^{* *}$ & $-0.056^{* *}$ & $-0.061^{* *}$ & 0.010 & 0.002 \\
BAME & $(0.022)$ & $(0.022)$ & $(0.024)$ & $(0.031)$ & $(0.033)$ \\
& 0.025 & $0.133^{* * *}$ & $0.123^{* * *}$ & $0.141^{* * *}$ & $0.142^{* * *}$ \\
Female & $(0.026)$ & $(0.038)$ & $(0.042)$ & $(0.043)$ & $(0.043)$ \\
& $0.124^{* * *}$ & $0.149^{* * *}$ & $0.158^{* * *}$ & $0.162^{* * *}$ & $0.162^{* * *}$ \\
BAME $\times$ Female & $(0.017)$ & $(0.018)$ & $(0.019)$ & $(0.020)$ & $(0.020)$ \\
& & $-0.180^{* * *}$ & $-0.201^{* * *}$ & $-0.215^{* * *}$ & $-0.216^{* * *}$ \\
F2F interview baseline & & $(0.051)$ & $(0.055)$ & $(0.056)$ & $(0.056)$ \\
& $0.038^{* *}$ & $0.038^{* *}$ & $0.044^{* *}$ & $0.047^{* *}$ & $0.047^{* *}$ \\
Household size & $(0.019)$ & $(0.019)$ & $(0.020)$ & $(0.021)$ & $(0.021)$ \\
& 0.010 & 0.010 & 0.012 & 0.013 & 0.013 \\
& $(0.008)$ & $(0.008)$ & $(0.009)$ & $(0.009)$ & $(0.009)$
\end{tabular}


Living with a partner

\begin{tabular}{|c|c|c|}
\hline-0.012 & -0.024 & -0.026 \\
\hline$(0.044)$ & $(0.045)$ & $(0.045)$ \\
\hline-0.048 & -0.041 & -0.042 \\
\hline$(0.055)$ & $(0.055)$ & $(0.055)$ \\
\hline 0.039 & 0.046 & 0.044 \\
\hline$(0.054)$ & $(0.055)$ & $(0.055)$ \\
\hline $0.083^{* *}$ & $0.088^{* * *}$ & $0.088^{* * *}$ \\
\hline$(0.033)$ & $(0.034)$ & $(0.034)$ \\
\hline 0.045 & 0.064 & 0.064 \\
\hline$(0.042)$ & $(0.041)$ & $(0.041)$ \\
\hline $0.091^{* * *}$ & $0.092^{* * *}$ & $0.091^{* * *}$ \\
\hline$(0.034)$ & $(0.035)$ & $(0.035)$ \\
\hline-0.005 & -0.006 & -0.006 \\
\hline$(0.051)$ & $(0.051)$ & $(0.051)$ \\
\hline $0.072^{* * *}$ & 0.041 & 0.040 \\
\hline$(0.027)$ & (0.028) & $(0.028)$ \\
\hline 0.029 & 0.016 & 0.016 \\
\hline$(0.034)$ & $(0.034)$ & $(0.034)$ \\
\hline 0.030 & 0.010 & 0.009 \\
\hline$(0.036)$ & $(0.037)$ & $(0.037)$ \\
\hline 0.010 & 0.002 & 0.002 \\
\hline \multirow[t]{19}{*}{$(0.028)$} & $(0.028)$ & $(0.028)$ \\
\hline & $0.085^{* *}$ & $0.084^{* *}$ \\
\hline & $(0.036)$ & $(0.036)$ \\
\hline & $-0.120^{*}$ & $-0.118^{*}$ \\
\hline & $(0.069)$ & $(0.069)$ \\
\hline & $0.092^{* * *}$ & $0.093^{* * *}$ \\
\hline & $(0.032)$ & $(0.032)$ \\
\hline & -0.020 & -0.019 \\
\hline & $(0.056)$ & $(0.056)$ \\
\hline & 0.073 & 0.074 \\
\hline & $(0.074)$ & $(0.074)$ \\
\hline & $-0.369 * * *$ & $-0.364 * * *$ \\
\hline & $(0.079)$ & $(0.079)$ \\
\hline & 0.147 & 0.147 \\
\hline & $(0.114)$ & $(0.114)$ \\
\hline & 0.003 & 0.003 \\
\hline & $(0.005)$ & $(0.005)$ \\
\hline & & -0.017 \\
\hline & & $(0.024)$ \\
\hline
\end{tabular}

Divorced or separated

Never married

0.039

London

$0.083^{* *}$

Wales

$(0.033)$

Scotland

$(0.042)$

Northern Ireland

(0.034)

BA or higher

$(0.051)$

Diploma or equiv.
(0.027)
0.029

A Level or equiv.

0.030

GCSE or equiv.

Self-employed

$0.085^{* *}$

(0.036)

Unemployed

Retired

$(0.032)$

House-keeper

Student

Disabled

$-0.369 * *$

$(0.079)$

0.147

$(0.114)$

Net personal income

0.003

COVID-19 high risk

\begin{tabular}{|c|c|c|c|c|c|}
\hline D counrod & 14,155 & $\begin{array}{c}14,155 \\
013\end{array}$ & $\begin{array}{r}12,380 \\
0\end{array}$ & $\begin{array}{c}12,000 \\
0201\end{array}$ & $\begin{array}{r}11,999 \\
0021\end{array}$ \\
\hline
\end{tabular}

Note: All regressions include month of (baseline) interview fixed effects. The reference categories are: British White, male, aged 65 or older, online interview in 2017-2019, interview in April in 2017-2019, widowed, living in England (excluding London), no qualification, employed, and COVID-19 non-high risk. Robust standard errors in parentheses. ${ }^{* * *} \mathrm{p}<0.01,{ }^{* *}$ $\mathrm{p}<0.05,{ }^{*} \mathrm{p}<0.1$ 


\subsubsection{Differences between BAME and British White}

While the patterns documented in Table 3 are informative, they could mask important heterogeneities, if only because our BAME indicator includes several groups. In Table 4 we unpack the BAME indicator in several groups: Other White, Mixed, Black, BIP (Bangladeshi, Indian and Pakistani), Chinese (and other Asian), Arab, and British White (reference category). This exercise needs to be taken with caution given the small sample sizes of these groups (see Table 2). With this limitation in mind, we proceed by carrying out the same analysis as before, but with more ethnic indicators and hence more interactions with the gender dummy variable.

As expected, the findings on variables other than ethnicity are essentially the same, both qualitatively and quantitatively. With regards to ethnicity, we find that the estimated BAME coefficient in column (1) of Table $3(0.025, \mathrm{SE}=0.026)$ masked substantial heterogeneity: column (1) of Table 4 reveals that individuals in the Bangladeshi, Indian and Pakistani (BIP) group experienced a larger increase (0.106 standard deviations, $\mathrm{SE}=0.044)$ in mental distress compared to the British White group. Individuals belonging to the Arab group appear to have suffered large deteriorations of mental health, but these are imprecisely estimated on account of the very small number of individuals in this group (see Table 2).

Considering the interaction between ethnicity and gender, column (2) of Table 4, indicates that the BIP group is behind the differential increase in mental distress among BAME individuals by gender documented in Table 3. For instance, focusing on the specification with all the control variables, we find that: (a) among males, BIP individuals experience a higher increase (0.259 SD, 95\% CI: $[0.136,0.382]$ ) in mental distress compared to British White individuals; (b) among females, BIP individuals experience a non-statistically significant lower increase (-0.03 SD, 95\% CI: [-0.164,0.105]) in mental distress compared to British White individuals; (c) among British White individuals, females experience a higher increase (0.162 SD, 95\% CI: [0.123,0.201]) in mental distress compared to men; (d) among BIP individuals, females experience a non-statistically significant lower increase (-0.127 SD, 95\% CI: [-0.300,0.047]) in mental distress compared to 
men. ${ }^{7}$ We cannot reject that the increase in mental distress among BIP individuals does not differ by gender: 0.259 SD [95\% CI: 0.136, 0.382] among males, 0.133 SD [95\% CI: $-0.004,0.269]$ among females. While the point estimates are remarkably different, the confidence intervals are quite wide. This is not a surprise, given that we are looking at gender gaps within a group of individuals which account for only $5 \%$ of the sample (see Table 2 ).

Table 4: OLS regressions of (standardised) changes in mental distress in the UKHLS from Wave 9 (20172019) to April 2020 on BAME groups and other characteristics

(1) (2)

\begin{tabular}{|c|c|c|c|c|c|}
\hline$<25$ & $\begin{array}{c}0.117^{* * *} \\
(0.043)\end{array}$ & $\begin{array}{c}0.118^{* * *} \\
(0.043)\end{array}$ & $\begin{array}{c}0.057 \\
(0.056)\end{array}$ & $\begin{array}{c}0.096 \\
(0.074)\end{array}$ & $\begin{array}{c}0.083 \\
(0.076)\end{array}$ \\
\hline $25-34$ & $\begin{array}{c}0.106^{* * *} \\
(0.032)\end{array}$ & $\begin{array}{c}0.107^{* * *} \\
(0.032)\end{array}$ & $\begin{array}{c}0.071^{*} \\
(0.036)\end{array}$ & $\begin{array}{c}0.147^{* * *} \\
(0.046)\end{array}$ & $\begin{array}{c}0.135^{* * *} \\
(0.049)\end{array}$ \\
\hline $35-44$ & $\begin{array}{c}0.003 \\
(0.030)\end{array}$ & $\begin{array}{c}0.002 \\
(0.030)\end{array}$ & $\begin{array}{l}-0.014 \\
(0.033)\end{array}$ & $\begin{array}{c}0.070^{*} \\
(0.042)\end{array}$ & $\begin{array}{c}0.059 \\
(0.045)\end{array}$ \\
\hline $45-54$ & $\begin{array}{c}-0.113^{* * *} \\
(0.025)\end{array}$ & $\begin{array}{c}-0.114^{* * *} \\
(0.025)\end{array}$ & $\begin{array}{c}-0.136^{* * * *} \\
(0.028)\end{array}$ & $\begin{array}{l}-0.053 \\
(0.038)\end{array}$ & $\begin{array}{l}-0.062 \\
(0.040)\end{array}$ \\
\hline $55-64$ & $\begin{array}{c}-0.052^{* *} \\
(0.022)\end{array}$ & $\begin{array}{c}-0.053^{* *} \\
(0.022)\end{array}$ & $\begin{array}{c}-0.059^{* *} \\
(0.024)\end{array}$ & $\begin{array}{c}0.010 \\
(0.031)\end{array}$ & $\begin{array}{c}0.002 \\
(0.033)\end{array}$ \\
\hline Other White & $\begin{array}{c}0.033 \\
(0.043)\end{array}$ & $\begin{array}{c}0.105 \\
(0.066)\end{array}$ & $\begin{array}{c}0.155^{*} \\
(0.080)\end{array}$ & $\begin{array}{c}0.156^{*} \\
(0.080)\end{array}$ & $\begin{array}{c}0.156^{*} \\
(0.080)\end{array}$ \\
\hline Mixed & $\begin{array}{c}-0.091 \\
(0.072)\end{array}$ & $\begin{array}{l}-0.119 \\
(0.104)\end{array}$ & $\begin{array}{c}-0.164 \\
(0.108)\end{array}$ & $\begin{array}{c}-0.138 \\
(0.114)\end{array}$ & $\begin{array}{c}-0.138 \\
(0.114)\end{array}$ \\
\hline Black & $\begin{array}{c}-0.108 \\
(0.070)\end{array}$ & $\begin{array}{c}-0.005 \\
(0.102)\end{array}$ & $\begin{array}{c}-0.041 \\
(0.109)\end{array}$ & $\begin{array}{c}-0.019 \\
(0.109)\end{array}$ & $\begin{array}{c}-0.017 \\
(0.109)\end{array}$ \\
\hline BIP & $\begin{array}{l}0.106^{* *} \\
(0.044)\end{array}$ & $\begin{array}{c}0.265^{* * *} \\
(0.059)\end{array}$ & $\begin{array}{c}0.225^{* * *} \\
(0.060)\end{array}$ & $\begin{array}{c}0.258^{* * *} \\
(0.063)\end{array}$ & $\begin{array}{c}0.259^{* * *} \\
(0.063)\end{array}$ \\
\hline Chinese & $\begin{array}{l}-0.018 \\
(0.084)\end{array}$ & $\begin{array}{c}0.078 \\
(0.109)\end{array}$ & $\begin{array}{c}0.034 \\
(0.116)\end{array}$ & $\begin{array}{c}0.034 \\
(0.115)\end{array}$ & $\begin{array}{c}0.034 \\
(0.115)\end{array}$ \\
\hline Arab & $\begin{array}{c}0.230 \\
(0.201)\end{array}$ & $\begin{array}{c}0.433 \\
(0.263)\end{array}$ & $\begin{array}{c}0.501^{*} \\
(0.285)\end{array}$ & $\begin{array}{c}0.493^{*} \\
(0.282)\end{array}$ & $\begin{array}{c}0.490^{*} \\
(0.282)\end{array}$ \\
\hline Female & $\begin{array}{c}0.125^{* * *} \\
(0.017)\end{array}$ & $\begin{array}{c}0.149^{* * *} \\
(0.018)\end{array}$ & $\begin{array}{c}0.157^{* * *} \\
(0.019)\end{array}$ & $\begin{array}{c}0.162^{* * *} \\
(0.020)\end{array}$ & $\begin{array}{c}0.162^{* * *} \\
(0.020)\end{array}$ \\
\hline Other White $\times$ Female & & $\begin{array}{l}-0.117 \\
(0.087)\end{array}$ & $\begin{array}{l}-0.183^{*} \\
(0.100)\end{array}$ & $\begin{array}{l}-0.185^{*} \\
(0.101)\end{array}$ & $\begin{array}{l}-0.185^{*} \\
(0.101)\end{array}$ \\
\hline Mixed $\times$ Female & & $\begin{array}{c}0.043 \\
(0.142)\end{array}$ & $\begin{array}{c}0.045 \\
(0.146)\end{array}$ & $\begin{array}{c}-0.020 \\
(0.153)\end{array}$ & $\begin{array}{l}-0.019 \\
(0.153)\end{array}$ \\
\hline Black $\times$ Female & & $\begin{array}{l}-0.160 \\
(0.137)\end{array}$ & $\begin{array}{l}-0.177 \\
(0.143)\end{array}$ & $\begin{array}{c}-0.177 \\
(0.141)\end{array}$ & $\begin{array}{l}-0.177 \\
(0.141)\end{array}$ \\
\hline BIP $\times$ Female & & $\begin{array}{c}-0.277^{* * *} \\
(0.084)\end{array}$ & $\begin{array}{c}-0.273^{* * *} \\
(0.086)\end{array}$ & $\begin{array}{c}-0.288^{* * * *} \\
(0.090)\end{array}$ & $\begin{array}{c}-0.289^{* * * *} \\
(0.090)\end{array}$ \\
\hline Chinese $\times$ Female & & $\begin{array}{l}-0.167 \\
(0.163)\end{array}$ & $\begin{array}{l}-0.154 \\
(0.181)\end{array}$ & $\begin{array}{l}-0.160 \\
(0.183)\end{array}$ & $\begin{array}{l}-0.161 \\
(0.183)\end{array}$ \\
\hline Arab $\times$ Female & & $\begin{array}{c}-0.453 \\
(0.394)\end{array}$ & $\begin{array}{l}-0.446 \\
(0.433)\end{array}$ & $\begin{array}{l}-0.466 \\
(0.434)\end{array}$ & $\begin{array}{l}-0.465 \\
(0.434)\end{array}$ \\
\hline
\end{tabular}

\footnotetext{
7 Table A2 in Appendix C uses an indicator for BIP vs. non-BIP individuals.
} 
F2F interview baseline

$\begin{array}{cc}0.038^{* *} & 0.038^{* *} \\ (0.019) & (0.019) \\ 0.007 & 0.007 \\ (0.008) & (0.008)\end{array}$

$0.046^{* *}$
$(0.020)$
0.011
$(0.009)$
-0.013
$(0.045)$
-0.046
$(0.055)$
0.044
$(0.054)$
$0.095^{* * *}$
$(0.033)$
0.045
$(0.042)$
$0.093^{* * *}$
$(0.034)$
-0.014
$(0.051)$
$0.074 * *$
$(0.027)$
0.032
$(0.034)$
0.032
$(0.036)$
0.014
$(0.028)$

$0.048^{* *}$

$0.048^{* *}$

Household size

Living with a partner

(0.021)

(0.021)

0.011

0.011

(0.009)

(0.009)

Divorced or separated

$-0.024$

$-0.026$

$(0.045)$

(0.045)

$-0.040$

$-0.041$

Never married

(0.055)

$(0.055)$

0.052

0.050

London

(0.055)

(0.055)

Wales

$(0.034)$

$0.099 * * *$

0.065

$(0.034)$

0.065

Scotland

(0.042)

$(0.042)$

$0.094^{* * *}$

$0.093^{* * *}$

Northern Ireland

(0.035)

$(0.035)$

$-0.012$

$(0.052)$

BA or higher

(0.052)

0.043

0.042

Diploma or equiv.

$(0.028)$

$(0.028)$

0.018

0.018

$(0.034)$

$(0.034)$

A Level or equiv.

0.011

0.010

GCSE or equiv.

(0.037)

$(0.037)$

0.005

0.005

Self-employed

(0.029)

$0.082^{* *}$

$(0.029)$

(0.036)

$0.081^{* *}$

Unemployed

$-0.119 *$

(0.036)

(0.069)

$-0.118^{*}$

Retired

$0.089^{* * *}$

(0.069)

$(0.032)$

$0.090^{* * *}$

House-keeper

$-0.024$

$(0.032)$

(0.056)

$-0.024$

0.073

$(0.056)$

Student

(0.074)

0.073

Disabled

$-0.366^{* * *}$

(0.074)

(0.078)

$-0.362^{* * *}$

Other

0.149

(0.079)

(0.115)

0.148

0.003

$(0.115)$

(0.005)

0.003

$(0.005)$

$-0.017$

(0.024)

\begin{tabular}{lccccc}
\hline $\mathrm{N}$ & 14,155 & 14,155 & 12,380 & 12,000 & 11,999 \\
$\mathrm{R}$-squared & 0.013 & 0.014 & 0.018 & 0.022 & 0.022 \\
\hline
\end{tabular}

Note: All regressions include month of (baseline) interview fixed effects. The reference categories are: British White, male, aged 65 or older, online interview in 2017-2019, interview in April in 2017-2019, widowed, living in England (excluding London), no qualification, employed, and COVID-19 non-high risk. Robust standard errors in parentheses. ${ }^{* * *}$ p $<0.01$, ${ }^{* *}$ $\mathrm{p}<0.05,{ }^{*} \mathrm{p}<0.1$

3.2. Changes in mental distress in the working population 


\subsubsection{Differences between BAME and British White}

We now shift our attention to the working population by focusing on individuals who are employed or self-employed. Hence, our longitudinal sample decreases from 14,155 individuals to 8,695 individuals.

Column (1) in Table 5 displays an estimated coefficient on BAME of 0.059 $(\mathrm{SE}=0.033)$, doubling in size that the one in column (1) of Table 3, 0.025 (SE = 0.026). ${ }^{8}$ We also find that working females have experienced a larger increase $(0.131 \mathrm{SD}, \mathrm{SE}=0.022)$ in mental distress compared to working males, a similar differential increase as the one for the general population documented in Table 3. Interestingly, among working individuals, the larger the household size, the larger the increase in mental distress is.

When introducing the interaction between the BAME and the female indicators, we obtain similar results as in Table 3. For instance, focusing on the estimates in column (5), we find that: (a) among males, BAME individuals experience a higher increase (0.196 SD, 95\% CI: [0.094, 0.298]) in mental distress compared to British White individuals; (b) among females, BAME individuals experience a non-statistically significant lower increase (-0.038 SD, 95\% CI: [$0.144,0.067]$ ) in mental distress compared to British White individuals; (c) among British White individuals, females experience a higher increase $(0.18$ SD, 95\% CI: $[0.128,0.232])$ in mental distress compared to men; (d) among BAME individuals, females experience a non-statistically significant lower increase (-0.054 SD, 95\% CI: $[-0.185,0.077])$ in mental distress compared to men. We cannot reject that the increase in mental distress among BAME individuals does not differ by gender: 0.196 SD [95\% CI: 0.094, 0.298] among males, 0.142 SD [95\% CI: 0.035, 0.248] among females.

The addition of sociodemographic variables makes household size to become an irrelevant predictor, at least in terms of statistical significance. ${ }^{9}$ Interestingly, we do not find differences in increases in mental distress by place of residence, at least not when we control for type of occupation, which actually

\footnotetext{
8 Table A3 in Appendix C uses an indicator for White vs. non-White individuals as Daly et al. (2020).

${ }_{9}^{9}$ Since we are focusing on working individuals, the employment status categories are just two: employed or self-employed.
} 
predicts changes in mental distress: individuals categorised as working in "small employers \& own account" occupations have experienced a larger increase $(0.189$ $\mathrm{SD}, \mathrm{SE}=0.066)$ in mental distress compared to individuals working in "managerial and professional" occupations. Table 5: OLS regressions of (standardised) changes in mental distress in the UKHLS from Wave 9 (2017-2019)
to April 2020 among working individuals (employed or self-employed) on BAME and other characteristics

\section{(1)}

$0.151^{*}$
$(0.081)$

$0.151^{* * *}$

$(0.048)$

0.041

$(0.046)$

$-0.068$

$(0.043)$

$55-64$

$-0.004$

(0.042)

$0.059^{*}$

(0.033)

Female

BAME $\times$ Female

F2F interview baseline

$(0.022)$

Household size

Living with a partner

Divorced or separated

Never married

London

Wales

Scotland

Northern Ireland

BA or higher

Diploma or equiv.

A Level or equiv.
(2)

(3)

(4)

(5)

\begin{tabular}{|c|c|c|c|}
\hline $0.151^{*}$ & 0.104 & $0.183^{*}$ & $0.179^{*}$ \\
\hline$(0.081)$ & $(0.092)$ & $(0.099)$ & $(0.101)$ \\
\hline $0.151^{* * *}$ & $0.116^{* *}$ & $0.171^{* * *}$ & $0.167^{* * *}$ \\
\hline$(0.048)$ & $(0.055)$ & $(0.061)$ & $(0.064)$ \\
\hline 0.039 & 0.025 & 0.086 & 0.083 \\
\hline$(0.046)$ & $(0.051)$ & $(0.057)$ & $(0.060)$ \\
\hline$-0.071^{*}$ & $-0.093^{* *}$ & -0.034 & -0.037 \\
\hline$(0.043)$ & $(0.048)$ & $(0.053)$ & $(0.055)$ \\
\hline-0.005 & -0.020 & 0.007 & 0.005 \\
\hline$(0.042)$ & $(0.046)$ & $(0.052)$ & $(0.054)$ \\
\hline $0.205^{* * *}$ & $0.204^{* * *}$ & $0.196^{* * *}$ & $0.196^{* * *}$ \\
\hline$(0.046)$ & $(0.050)$ & $(0.052)$ & $(0.052)$ \\
\hline $0.170^{* * *}$ & $0.179 * * *$ & $0.180^{* * *}$ & $0.180^{* * *}$ \\
\hline$(0.023)$ & $(0.025)$ & $(0.026)$ & $(0.026)$ \\
\hline $0.257^{* * *}$ & $-0.276^{* * *}$ & $-0.234^{* * *}$ & $-0.234^{* * *}$ \\
\hline$(0.064)$ & $(0.068)$ & $(0.071)$ & $(0.071)$ \\
\hline $0.060^{* *}$ & $0.058^{* *}$ & $0.059 * *$ & $0.059^{* *}$ \\
\hline$(0.024)$ & $(0.026)$ & $(0.027)$ & $(0.027)$ \\
\hline $0.017^{*}$ & $0.020^{* *}$ & 0.014 & 0.014 \\
\hline \multirow[t]{20}{*}{$(0.009)$} & $(0.010)$ & $(0.011)$ & $(0.011)$ \\
\hline & 0.046 & -0.017 & -0.017 \\
\hline & $(0.087)$ & $(0.091)$ & $(0.091)$ \\
\hline & -0.007 & -0.035 & -0.035 \\
\hline & $(0.095)$ & $(0.099)$ & (0.099) \\
\hline & 0.110 & 0.042 & 0.041 \\
\hline & $(0.094)$ & $(0.099)$ & $(0.099)$ \\
\hline & $0.071^{*}$ & 0.040 & 0.040 \\
\hline & $(0.042)$ & $(0.044)$ & $(0.044)$ \\
\hline & 0.081 & 0.046 & 0.046 \\
\hline & $(0.051)$ & $(0.054)$ & $(0.054)$ \\
\hline & $0.082^{*}$ & 0.068 & 0.068 \\
\hline & $(0.043)$ & $(0.045)$ & $(0.045)$ \\
\hline & -0.050 & 0.003 & 0.003 \\
\hline & $(0.064)$ & $(0.062)$ & $(0.062)$ \\
\hline & -0.009 & -0.001 & -0.002 \\
\hline & $(0.037)$ & $(0.041)$ & $(0.041)$ \\
\hline & -0.052 & -0.040 & -0.040 \\
\hline & $(0.046)$ & $(0.049)$ & $(0.049)$ \\
\hline & -0.063 & -0.073 & -0.073 \\
\hline
\end{tabular}


GCSE or equiv.

Self-employed

Net personal income

Intermediate

Small employers \& own account

Semi-routine \& routine

COVID-19 high risk

\begin{tabular}{llllll}
\hline $\mathrm{N}$ & 8,695 & 8,695 & 7,729 & 7,022 & 7,021 \\
$\mathrm{R}$-squared & 0.014 & 0.016 & 0.020 & 0.021 & 0.021 \\
\hline
\end{tabular}

Note: All regressions include month of (baseline) interview fixed effects. The reference categories are: British White, male, aged 65 or older, online interview in 2017-2019, interview in April in 2017-2019, widowed, living in England (excluding London), no qualification, employed, Management and Professional, COVID-19 non-high risk. Robust standard errors in parentheses. ${ }^{* * *} \mathrm{p}<0.01,{ }^{* *} \mathrm{p}<0.05,{ }^{*} \mathrm{p}<0.1$

\subsubsection{Differences among ethnic groups}

Table 6 contains the analysis using different BAME categories and reveals that the results in Table 5 are driven by Bangladeshi, Indian and Pakistani working individuals. ${ }^{10}$ Once again, it is important to be aware of the small samples involved in this analysis. This is particularly important here because the sample is further restricted by focusing on employed and self-employed individuals.

${ }^{10}$ Table A4 in the Appendix C uses an indicator for BIP vs. non-BIP individuals. 
Table 6: OLS regressions of (standardised) changes in mental distress in the UKHLS from Wave 9 (20172019) to April 2020among working individuals (employed or self-employed) on BAME groups and other characteristics

(1)

\begin{tabular}{|c|c|c|c|c|c|}
\hline & (1) & $(Z)$ & (3) & (4) & (5) \\
\hline \multirow[t]{2}{*}{$<25$} & $0.146^{*}$ & $0.147^{*}$ & 0.095 & $0.173^{*}$ & $0.167^{*}$ \\
\hline & $(0.081)$ & $(0.081)$ & $(0.092)$ & $(0.099)$ & $(0.101)$ \\
\hline \multirow[t]{2}{*}{$25-34$} & $0.150^{* * *}$ & $0.149^{* * *}$ & $0.113^{* *}$ & $0.168^{* * *}$ & $0.163^{* *}$ \\
\hline & $(0.048)$ & $(0.048)$ & $(0.055)$ & $(0.061)$ & $(0.064)$ \\
\hline \multirow[t]{2}{*}{$35-44$} & 0.042 & 0.038 & 0.022 & 0.083 & 0.078 \\
\hline & $(0.046)$ & $(0.046)$ & $(0.051)$ & $(0.057)$ & $(0.060)$ \\
\hline \multirow[t]{2}{*}{$45-54$} & -0.065 & -0.068 & $-0.092^{*}$ & -0.032 & -0.037 \\
\hline & $(0.043)$ & $(0.043)$ & $(0.048)$ & $(0.053)$ & $(0.056)$ \\
\hline \multirow[t]{2}{*}{$55-64$} & -0.002 & -0.004 & -0.019 & 0.009 & 0.005 \\
\hline & $(0.042)$ & $(0.042)$ & $(0.046)$ & $(0.052)$ & $(0.054)$ \\
\hline \multirow[t]{2}{*}{ Other White } & 0.037 & $0.183^{* *}$ & $0.233^{* *}$ & 0.164 & 0.164 \\
\hline & $(0.053)$ & $(0.085)$ & $(0.097)$ & $(0.100)$ & $(0.100)$ \\
\hline \multirow[t]{2}{*}{ Mixed } & -0.102 & -0.105 & -0.129 & -0.108 & -0.108 \\
\hline & $(0.087)$ & $(0.125)$ & $(0.132)$ & $(0.133)$ & $(0.134)$ \\
\hline \multirow[t]{2}{*}{ Black } & -0.042 & 0.111 & 0.101 & 0.111 & 0.112 \\
\hline & $(0.075)$ & $(0.097)$ & $(0.104)$ & $(0.111)$ & $(0.111)$ \\
\hline \multirow[t]{2}{*}{ BIP } & $0.202^{* * *}$ & $0.362^{* * *}$ & $0.334^{* * *}$ & $0.359^{* * *}$ & $0.360^{* * *}$ \\
\hline & $(0.059)$ & $(0.073)$ & $(0.074)$ & $(0.077)$ & $(0.077)$ \\
\hline \multirow[t]{2}{*}{ Chinese } & -0.032 & 0.052 & 0.017 & -0.005 & -0.005 \\
\hline & $(0.108)$ & $(0.125)$ & $(0.134)$ & $(0.127)$ & $(0.127)$ \\
\hline \multirow[t]{2}{*}{ Arab } & 0.308 & $0.577^{*}$ & $0.572^{*}$ & $0.576^{*}$ & $0.575^{*}$ \\
\hline & $(0.249)$ & $(0.301)$ & $(0.324)$ & $(0.328)$ & $(0.328)$ \\
\hline \multirow[t]{2}{*}{ Female } & $0.134^{* * *}$ & $0.170^{* * *}$ & $0.179 * * *$ & $0.180^{* * *}$ & $0.180^{* * *}$ \\
\hline & $(0.022)$ & $(0.023)$ & $(0.025)$ & $(0.026)$ & $(0.026)$ \\
\hline \multirow[t]{2}{*}{ Other White $\times$ Female } & & $-0.238^{* *}$ & $-0.304^{* *}$ & -0.198 & -0.198 \\
\hline & & $(0.108)$ & $(0.121)$ & $(0.125)$ & $(0.125)$ \\
\hline \multirow[t]{2}{*}{ Mixed $\times$ Female } & & 0.004 & -0.021 & -0.039 & -0.039 \\
\hline & & $(0.173)$ & $(0.179)$ & $(0.184)$ & $(0.184)$ \\
\hline \multirow[t]{2}{*}{ Black $\times$ Female } & & $-0.240^{*}$ & $-0.274^{*}$ & -0.247 & -0.248 \\
\hline & & $(0.142)$ & $(0.147)$ & $(0.161)$ & $(0.161)$ \\
\hline \multirow[t]{2}{*}{ BIP $\times$ Female } & & $-0.319 * * *$ & $-0.309 * * *$ & $-0.286^{* *}$ & $-0.286^{* *}$ \\
\hline & & $(0.116)$ & $(0.118)$ & $(0.125)$ & $(0.125)$ \\
\hline \multirow[t]{2}{*}{ Chinese $\times$ Female } & & -0.158 & -0.131 & -0.103 & -0.104 \\
\hline & & $(0.213)$ & $(0.239)$ & $(0.246)$ & $(0.247)$ \\
\hline \multirow[t]{2}{*}{ Arab $\times$ Female } & & $-0.829 *$ & -0.612 & -0.175 & -0.175 \\
\hline & & $(0.446)$ & $(0.560)$ & $(0.514)$ & $(0.514)$ \\
\hline \multirow[t]{2}{*}{ F2F interview baseline } & $0.059 * *$ & $0.059^{* *}$ & $0.058^{* *}$ & $0.058 * *$ & $0.058^{* *}$ \\
\hline & $(0.024)$ & $(0.024)$ & $(0.026)$ & $(0.027)$ & $(0.027)$ \\
\hline \multirow[t]{2}{*}{ Household size } & 0.014 & 0.013 & $0.017^{*}$ & 0.011 & 0.011 \\
\hline & $(0.009)$ & $(0.009)$ & $(0.010)$ & $(0.010)$ & $(0.010)$ \\
\hline \multirow[t]{2}{*}{ Living with a partner } & & & 0.047 & -0.016 & -0.016 \\
\hline & & & $(0.087)$ & $(0.091)$ & $(0.091)$ \\
\hline \multirow[t]{2}{*}{ Divorced or separated } & & & -0.007 & -0.034 & -0.035 \\
\hline & & & $(0.095)$ & $(0.099)$ & $(0.099)$ \\
\hline \multirow[t]{2}{*}{ Never married } & & & 0.116 & 0.050 & 0.049 \\
\hline & & & $(0.094)$ & $(0.099)$ & $(0.099)$ \\
\hline
\end{tabular}


London

$\begin{array}{ccc}0.078^{*} & 0.047 & 0.047 \\ (0.042) & (0.044) & (0.044) \\ 0.084^{*} & 0.049 & 0.050 \\ (0.051) & (0.054) & (0.054) \\ 0.086^{* *} & 0.073 & 0.073 \\ (0.043) & (0.045) & (0.045) \\ -0.052 & 0.007 & 0.008 \\ (0.064) & (0.062) & (0.062) \\ -0.007 & 0.002 & 0.002 \\ (0.037) & (0.041) & (0.041) \\ -0.050 & -0.038 & -0.038 \\ (0.047) & (0.049) & (0.049) \\ -0.065 & -0.076 & -0.077 \\ (0.049) & (0.052) & (0.052) \\ -0.045 & -0.049 & -0.049 \\ (0.039) & (0.040) & (0.040) \\ & -0.010 & -0.010 \\ & (0.057) & (0.057) \\ & 0.003 & 0.003 \\ & (0.006) & (0.006) \\ & -0.023 & -0.023 \\ & (0.038) & (0.038) \\ & 0.191 * * * & 0.191^{* * *} \\ & (0.065) & (0.065) \\ & 0.058 & 0.058 \\ & (0.051) & (0.051) \\ & 0.009 & 0.009 \\ & (0.035) & (0.035) \\ & & -0.008 \\ & & (0.030)\end{array}$

Wales

Scotland

Northern Ireland

$-0.052$

BA or higher

Diploma or equiv.

$$
-0.007
$$

A Level or equiv.

$(0.047)$

GCSE or equiv.

Self-employed

Net personal income

0.003

Intermediate

Small employers \& own account

$(0.065)$

Lower supervisory \& technical

0.058

Semi-routine $\&$ routine

0.009

7,021

\begin{tabular}{llllll}
\hline $\mathrm{N}$ & 8,695 & 8,695 & 7,729 & 7,022 & 7,021 \\
$\mathrm{R}$-squared & 0.016 & 0.018 & 0.022 & 0.023 & 0.023 \\
\hline
\end{tabular}

Note: All regressions include month of (baseline) interview fixed effects. The reference categories are: British White, male, aged 65 or older, online interview in 2017-2019, interview in April in 2017-2019, widowed, living in England (excluding London), no qualification, employed, Management and Professional, COVID-19 non-high risk. Robust standard errors in parentheses. ${ }^{* * *} \mathrm{p}<0.01,{ }^{* *} \mathrm{p}<0.05,{ }^{*} \mathrm{p}<0.1$

\subsection{Changes in the likelihood of having mental health problems}

\subsubsection{General population}

In Table 7 we study potential predictors of changes in the likelihood of having mental health problems. As explained in section 2, and following the recent work by Daly et al. (2020), having mental health problems is defined as having a GHQ 'caseness' score $\geq 3$ (i.e., having at least 3 out of 12 symptoms). Column (1) shows that women have experienced a higher increase (7.5 pp) in mental health problems compared to men, that younger individuals (25-34 years old) have experienced a higher increase in mental health problems compared to individuals 
aged 65 or above, that there are no mode of survey administration effects, that household size is an irrelevant predictor of changes in mental health status, and that both BAME and British White individuals have experienced similar increases in the likelihood of having mental health problems.

We also find that individuals with a university degree have also experienced a higher increase (5 pp) in mental health problems compared to those with no qualification, that neither marital status nor net personal income predicts changes in mental health problems, and that both COVID-19 high-risk individuals and nonhigh risk individuals have experienced similar increases in mental health problems. All these findings are consistent with Daly et al. (2020). ${ }^{11}$ In addition, we find a larger increase (4.3 pp) in mental health problems among individuals living in London compared to those living in England (London excluded), and lower increases (1.2 pp and $1.6 \mathrm{pp}$ ) among unemployed and disabled individuals compared to those of employed individuals.

Once we allow for gender to interact with ethnicity in predicting changes in mental health, we find similar patterns as the ones documented in Table 3: (a) among males, BAME individuals experience a higher increase (5.3 pp, 95\% CI: $[0.8,9.8])$ in mental health problems compared to British White individuals; (b) among females, BAME individuals experience a lower increase (-4.9 pp, 95\% CI: [9.0,-0.8]) in mental health problems compared to British White individuals; (c) among British White individuals, females experience a higher increase (9.2 pp, 95\% CI: [7.0,11.4]) in mental health problems compared to men; (d) among BAME individuals, females experience a non-statistically significant lower increase (-1.0 pp, 95\% CI: $[-6.4,4.5])$ in mental health problems compared to men.

\footnotetext{
11 Comparing White versus non-White individuals, as in Daly et al. (2020), we find similar results in column (1) of Table A5 (see Appendix C).
} 
Table 7: OLS regressions of percentage point changes in likelihood of having mental health problems in the UKHLS from Wave 9 (2017-2019) to April 2020 on BAME and other characteristics

(1) (2)

\begin{tabular}{|c|c|c|c|c|c|}
\hline & (1) & (L) & (3) & (4) & (5) \\
\hline \multirow[t]{2}{*}{$<25$} & 0.015 & 0.015 & 0.004 & 0.017 & 0.020 \\
\hline & $(0.023)$ & $(0.023)$ & $(0.030)$ & $(0.040)$ & $(0.041)$ \\
\hline \multirow[t]{2}{*}{$25-34$} & $0.059 * * *$ & $0.060^{* * *}$ & $0.042^{* *}$ & $0.073^{* * *}$ & $0.076^{* * *}$ \\
\hline & $(0.018)$ & $(0.018)$ & $(0.020)$ & $(0.026)$ & $(0.027)$ \\
\hline \multirow[t]{2}{*}{$35-44$} & 0.019 & 0.019 & 0.010 & $0.042^{*}$ & $0.045^{*}$ \\
\hline & $(0.016)$ & $(0.016)$ & $(0.018)$ & $(0.024)$ & $(0.025)$ \\
\hline \multirow[t]{2}{*}{$45-54$} & -0.010 & -0.011 & -0.016 & 0.015 & 0.018 \\
\hline & $(0.014)$ & $(0.014)$ & $(0.016)$ & $(0.022)$ & $(0.023)$ \\
\hline \multirow[t]{2}{*}{$55-64$} & -0.017 & -0.017 & -0.015 & 0.010 & 0.012 \\
\hline & $(0.013)$ & $(0.013)$ & $(0.014)$ & $(0.019)$ & $(0.019)$ \\
\hline \multirow[t]{2}{*}{ BAME } & -0.002 & $0.044^{* *}$ & $0.041^{*}$ & $0.053^{* *}$ & $0.053^{* *}$ \\
\hline & $(0.014)$ & $(0.020)$ & $(0.022)$ & $(0.023)$ & $(0.023)$ \\
\hline \multirow[t]{2}{*}{ Female } & $0.075^{* * *}$ & $0.086^{* * *}$ & $0.089 * * *$ & $0.092^{* * *}$ & $0.092^{* * *}$ \\
\hline & $(0.009)$ & $(0.010)$ & $(0.011)$ & $(0.011)$ & $(0.011)$ \\
\hline \multirow[t]{2}{*}{ BAME $\times$ Female } & & $-0.077^{* * *}$ & $-0.094^{* * *}$ & $-0.102^{* * *}$ & $-0.102^{* * *}$ \\
\hline & & $(0.026)$ & $(0.029)$ & $(0.029)$ & $(0.029)$ \\
\hline \multirow{2}{*}{ F2F interview baseline } & 0.004 & 0.004 & 0.009 & 0.011 & 0.011 \\
\hline & $(0.010)$ & $(0.010)$ & $(0.011)$ & $(0.011)$ & $(0.011)$ \\
\hline \multirow[t]{2}{*}{ Household size } & 0.005 & 0.005 & 0.004 & 0.004 & 0.004 \\
\hline & $(0.004)$ & $(0.004)$ & $(0.005)$ & $(0.005)$ & $(0.005)$ \\
\hline \multirow[t]{2}{*}{ Living with a partner } & & & -0.002 & -0.009 & -0.008 \\
\hline & & & $(0.029)$ & $(0.029)$ & $(0.029)$ \\
\hline \multirow[t]{2}{*}{ Divorced or separated } & & & -0.012 & -0.007 & -0.006 \\
\hline & & & $(0.033)$ & $(0.034)$ & $(0.034)$ \\
\hline \multirow[t]{2}{*}{ Never married } & & & 0.008 & 0.013 & 0.013 \\
\hline & & & $(0.033)$ & $(0.034)$ & $(0.034)$ \\
\hline \multirow[t]{2}{*}{ London } & & & $0.043^{* *}$ & $0.043^{* *}$ & $0.043^{* *}$ \\
\hline & & & $(0.018)$ & $(0.019)$ & $(0.019)$ \\
\hline \multirow[t]{2}{*}{ Wales } & & & 0.004 & 0.009 & 0.009 \\
\hline & & & $(0.024)$ & $(0.024)$ & $(0.024)$ \\
\hline \multirow[t]{2}{*}{ Scotland } & & & $0.034^{*}$ & 0.029 & 0.029 \\
\hline & & & $(0.019)$ & $(0.019)$ & $(0.019)$ \\
\hline \multirow[t]{2}{*}{ Northern Ireland } & & & 0.014 & 0.010 & 0.010 \\
\hline & & & $(0.028)$ & $(0.028)$ & $(0.028)$ \\
\hline \multirow[t]{2}{*}{ BA or higher } & & & $0.063^{* * *}$ & $0.050 * * *$ & $0.050 * * *$ \\
\hline & & & $(0.015)$ & $(0.015)$ & $(0.015)$ \\
\hline \multirow[t]{2}{*}{ Diploma or equiv. } & & & $0.034^{*}$ & 0.028 & 0.028 \\
\hline & & & $(0.018)$ & $(0.019)$ & $(0.019)$ \\
\hline \multirow[t]{2}{*}{ A Level or equiv. } & & & $0.037^{*}$ & 0.027 & 0.027 \\
\hline & & & $(0.019)$ & $(0.020)$ & $(0.020)$ \\
\hline \multirow[t]{2}{*}{ GCSE or equiv. } & & & 0.010 & 0.007 & 0.007 \\
\hline & & & $(0.015)$ & $(0.015)$ & $(0.015)$ \\
\hline Self-employed & & & & 0.024 & 0.024 \\
\hline & & & & $(0.019)$ & $(0.019)$ \\
\hline Unemployed & & & & $-0.123^{* * *}$ & $-0.123^{* * *}$ \\
\hline & & & & $(0.033)$ & $(0.033)$ \\
\hline Retired & & & & 0.031 & 0.031 \\
\hline & & & & $(0.019)$ & $(0.019)$ \\
\hline House-keeper & & & & 0.006 & 0.005 \\
\hline
\end{tabular}




$\begin{array}{lcc} & & (0.028) \\ \text { Student } & (0.028) & 0.032 \\ & 0.032 & (0.039) \\ \text { Disabled } & (0.039) & -0.162^{* * *} \\ & -0.160^{* * *} & (0.037) \\ \text { Other } & (0.037) & 0.062 \\ & 0.062 & (0.062) \\ \text { Net personal income } & (0.062) & 0.002 \\ \text { COVID-19 high risk } & 0.002 & (0.003) \\ & (0.003) & 0.004 \\ & & (0.013)\end{array}$

\begin{tabular}{lccccc}
\hline $\mathrm{N}$ & 14,155 & 14,155 & 12,380 & 12,000 & 11,999 \\
$\mathrm{R}$-squared & 0.008 & 0.008 & 0.011 & 0.015 & 0.015 \\
\hline
\end{tabular}

Note: All regressions include month of (baseline) interview fixed effects. The reference categories are: British White, male, aged 65 or older, online interview in 2017-2019, interview in April in 2017-2019, widowed, living in England (excluding London), no qualification, employed, and COVID-19 non-high risk. Robust standard errors in parentheses. ${ }^{* * *} \mathrm{p}<0.01$, ${ }^{* *}$ $\mathrm{p}<0.05,{ }^{*} \mathrm{p}<0.1$

In Table 8 we redo the analysis in Table 7 but replacing the BAME indicators with several ethnic group indicators. As before, the estimates in Table 8 show that the differences between BAME and British White appear to be driven by "Bangladeshi, Indian and Pakistani". 12

\section{Table 8: OLS regressions of percentage point changes in likelihood of having mental health problems in the UKHLS from Wave 9 (2017-2019) to April 2020 on BAME groups and other characteristics}

\begin{tabular}{|c|c|c|c|c|c|}
\hline & $(1)$ & $(2)$ & $(3)$ & (4) & $(5)$ \\
\hline \multirow[t]{2}{*}{$<25$} & 0.016 & 0.017 & 0.002 & 0.015 & 0.018 \\
\hline & $(0.023)$ & $(0.023)$ & $(0.030)$ & $(0.040)$ & $(0.041)$ \\
\hline \multirow[t]{2}{*}{$25-34$} & $0.060 * * *$ & $0.061^{* * *}$ & $0.042^{* *}$ & $0.072^{* * *}$ & $0.075^{* * *}$ \\
\hline & $(0.018)$ & $(0.018)$ & $(0.020)$ & $(0.026)$ & $(0.027)$ \\
\hline \multirow[t]{2}{*}{$35-44$} & 0.021 & 0.020 & 0.010 & $0.041^{*}$ & $0.044^{*}$ \\
\hline & $(0.016)$ & $(0.016)$ & $(0.018)$ & $(0.024)$ & $(0.025)$ \\
\hline \multirow[t]{2}{*}{$45-54$} & -0.008 & -0.009 & -0.015 & 0.016 & 0.018 \\
\hline & $(0.014)$ & $(0.014)$ & $(0.016)$ & $(0.022)$ & $(0.023)$ \\
\hline \multirow[t]{2}{*}{$55-64$} & -0.016 & -0.016 & -0.014 & 0.010 & 0.012 \\
\hline & $(0.013)$ & $(0.013)$ & $(0.014)$ & $(0.019)$ & $(0.019)$ \\
\hline \multirow[t]{2}{*}{ Other White } & 0.014 & 0.031 & 0.056 & 0.065 & 0.065 \\
\hline & $(0.023)$ & $(0.034)$ & $(0.043)$ & $(0.044)$ & $(0.044)$ \\
\hline \multirow[t]{2}{*}{ Mixed } & -0.048 & -0.031 & -0.049 & -0.030 & -0.030 \\
\hline & $(0.039)$ & $(0.055)$ & $(0.057)$ & $(0.061)$ & $(0.061)$ \\
\hline \multirow[t]{2}{*}{ Black } & $-0.062^{*}$ & -0.013 & -0.020 & -0.003 & -0.004 \\
\hline & $(0.034)$ & $(0.049)$ & $(0.052)$ & $(0.052)$ & $(0.052)$ \\
\hline \multirow[t]{2}{*}{ BIP } & 0.025 & $0.105^{* * *}$ & $0.086^{* * *}$ & $0.102^{* * *}$ & $0.101^{* * *}$ \\
\hline & $(0.022)$ & $(0.030)$ & $(0.032)$ & $(0.033)$ & $(0.033)$ \\
\hline
\end{tabular}

12 Table A6 in Appendix C uses an indicator for BIP vs. non-BIP individuals. 


\begin{tabular}{|c|c|c|c|c|c|}
\hline Chinese & $\begin{array}{c}-0.058 \\
(0.045)\end{array}$ & $\begin{array}{c}-0.037 \\
(0.067)\end{array}$ & $\begin{array}{c}-0.077 \\
(0.072)\end{array}$ & $\begin{array}{c}-0.076 \\
(0.071)\end{array}$ & $\begin{array}{c}-0.076 \\
(0.071)\end{array}$ \\
\hline Arab & $\begin{array}{c}0.168 \\
(0.109)\end{array}$ & $\begin{array}{c}0.168 \\
(0.158)\end{array}$ & $\begin{array}{c}0.227 \\
(0.143)\end{array}$ & $\begin{array}{c}0.230 \\
(0.140)\end{array}$ & $\begin{array}{c}0.231^{*} \\
(0.140)\end{array}$ \\
\hline Female & $\begin{array}{c}0.076^{* * *} \\
(0.009)\end{array}$ & $\begin{array}{c}0.086^{* * *} \\
(0.010)\end{array}$ & $\begin{array}{c}0.089 * * * \\
(0.011)\end{array}$ & $\begin{array}{c}0.092^{* * *} \\
(0.011)\end{array}$ & $\begin{array}{c}0.092^{* * *} \\
(0.011)\end{array}$ \\
\hline Other White $\times$ Female & & $\begin{array}{c}-0.027 \\
(0.045)\end{array}$ & $\begin{array}{c}-0.072 \\
(0.055)\end{array}$ & $\begin{array}{c}-0.082 \\
(0.055)\end{array}$ & $\begin{array}{c}-0.082 \\
(0.055)\end{array}$ \\
\hline Mixed $\times$ Female & & $\begin{array}{c}-0.028 \\
(0.076)\end{array}$ & $\begin{array}{c}-0.024 \\
(0.079)\end{array}$ & $\begin{array}{l}-0.046 \\
(0.084)\end{array}$ & $\begin{array}{c}-0.046 \\
(0.084)\end{array}$ \\
\hline Black $\times$ Female & & $\begin{array}{c}-0.076 \\
(0.067)\end{array}$ & $\begin{array}{c}-0.095 \\
(0.069)\end{array}$ & $\begin{array}{c}-0.108 \\
(0.069)\end{array}$ & $\begin{array}{c}-0.108 \\
(0.069)\end{array}$ \\
\hline BIP $\times$ Female & & $\begin{array}{c}-0.139 * * * \\
(0.041)\end{array}$ & $\begin{array}{c}-0.138^{* * *} \\
(0.043)\end{array}$ & $\begin{array}{c}-0.142^{* * *} \\
(0.045)\end{array}$ & $\begin{array}{c}-0.141^{* * *} \\
(0.045)\end{array}$ \\
\hline Chinese $\times$ Female & & $\begin{array}{c}-0.037 \\
(0.091)\end{array}$ & $\begin{array}{c}-0.025 \\
(0.098)\end{array}$ & $\begin{array}{c}-0.021 \\
(0.099)\end{array}$ & $\begin{array}{c}-0.021 \\
(0.099)\end{array}$ \\
\hline Arab $\times$ Female & & $\begin{array}{c}0.003 \\
(0.214)\end{array}$ & $\begin{array}{c}-0.000 \\
(0.214)\end{array}$ & $\begin{array}{c}-0.021 \\
(0.212)\end{array}$ & $\begin{array}{c}-0.021 \\
(0.212)\end{array}$ \\
\hline F2F interview baseline & $\begin{array}{c}0.005 \\
(0.010)\end{array}$ & $\begin{array}{c}0.005 \\
(0.010)\end{array}$ & $\begin{array}{c}0.010 \\
(0.011)\end{array}$ & $\begin{array}{c}0.012 \\
(0.011)\end{array}$ & $\begin{array}{c}0.012 \\
(0.011)\end{array}$ \\
\hline Household size & $\begin{array}{c}0.004 \\
(0.004)\end{array}$ & $\begin{array}{c}0.004 \\
(0.004)\end{array}$ & $\begin{array}{c}0.004 \\
(0.005)\end{array}$ & $\begin{array}{c}0.003 \\
(0.005)\end{array}$ & $\begin{array}{c}0.003 \\
(0.005)\end{array}$ \\
\hline Living with a partner & & & $\begin{array}{c}-0.003 \\
(0.029)\end{array}$ & $\begin{array}{c}-0.009 \\
(0.029)\end{array}$ & $\begin{array}{c}-0.008 \\
(0.029)\end{array}$ \\
\hline Divorced or separated & & & $\begin{array}{c}-0.012 \\
(0.033)\end{array}$ & $\begin{array}{c}-0.006 \\
(0.034)\end{array}$ & $\begin{array}{c}-0.006 \\
(0.034)\end{array}$ \\
\hline Never married & & & $\begin{array}{c}0.011 \\
(0.033)\end{array}$ & $\begin{array}{c}0.016 \\
(0.034)\end{array}$ & $\begin{array}{c}0.016 \\
(0.034)\end{array}$ \\
\hline London & & & $\begin{array}{c}0.049^{* * *} \\
(0.019)\end{array}$ & $\begin{array}{l}0.048^{* *} \\
(0.019)\end{array}$ & $\begin{array}{l}0.048^{* *} \\
(0.019)\end{array}$ \\
\hline Wales & & & $\begin{array}{c}0.004 \\
(0.024)\end{array}$ & $\begin{array}{c}0.009 \\
(0.024)\end{array}$ & $\begin{array}{c}0.009 \\
(0.024)\end{array}$ \\
\hline Scotland & & & $\begin{array}{c}0.035^{*} \\
(0.019)\end{array}$ & $\begin{array}{c}0.030 \\
(0.019)\end{array}$ & $\begin{array}{c}0.030 \\
(0.019)\end{array}$ \\
\hline Northern Ireland & & & $\begin{array}{c}0.008 \\
(0.028)\end{array}$ & $\begin{array}{c}0.005 \\
(0.029)\end{array}$ & $\begin{array}{c}0.005 \\
(0.029)\end{array}$ \\
\hline BA or higher & & & $\begin{array}{c}0.064^{* * * *} \\
(0.015)\end{array}$ & $\begin{array}{c}0.051^{* * *} \\
(0.015)\end{array}$ & $\begin{array}{c}0.052^{* * *} \\
(0.015)\end{array}$ \\
\hline Diploma or equiv. & & & $\begin{array}{c}0.035^{*} \\
(0.018)\end{array}$ & $\begin{array}{c}0.028 \\
(0.019)\end{array}$ & $\begin{array}{c}0.028 \\
(0.019)\end{array}$ \\
\hline A Level or equiv. & & & $\begin{array}{c}0.037^{*} \\
(0.019)\end{array}$ & $\begin{array}{c}0.028 \\
(0.020)\end{array}$ & $\begin{array}{c}0.028 \\
(0.020)\end{array}$ \\
\hline GCSE or equiv. & & & $\begin{array}{c}0.012 \\
(0.015)\end{array}$ & $\begin{array}{c}0.008 \\
(0.015)\end{array}$ & $\begin{array}{c}0.008 \\
(0.015)\end{array}$ \\
\hline Self-employed & & & & $\begin{array}{c}0.023 \\
(0.019)\end{array}$ & $\begin{array}{c}0.023 \\
(0.019)\end{array}$ \\
\hline Unemployed & & & & $\begin{array}{c}-0.122^{* * *} \\
(0.033)\end{array}$ & $\begin{array}{c}-0.123^{* * *} \\
(0.033)\end{array}$ \\
\hline Retired & & & & $\begin{array}{c}0.030 \\
(0.019)\end{array}$ & $\begin{array}{c}0.029 \\
(0.019)\end{array}$ \\
\hline House-keeper & & & & $\begin{array}{c}0.004 \\
(0.029)\end{array}$ & $\begin{array}{c}0.003 \\
(0.029)\end{array}$ \\
\hline Student & & & & $\begin{array}{c}0.030 \\
(0.039)\end{array}$ & $\begin{array}{c}0.030 \\
(0.039)\end{array}$ \\
\hline
\end{tabular}


Disabled

Other

Net personal income

COVID-19 high risk

\begin{tabular}{|c|c|c|c|c|c|}
\hline $\mathrm{N}$ & 14,155 & 14,155 & 12,380 & 12,000 & $\begin{array}{c}11,999 \\
0.17\end{array}$ \\
\hline R-squared & 0.009 & 0.009 & 0.013 & 0.017 & 0.017 \\
\hline
\end{tabular}

Note: All regressions include month of (baseline) interview fixed effects. The reference categories are: British White, male, aged 65 or older, online interview in 2017-2019, interview in April in 2017-2019, widowed, living in England (excluding London), no qualification, employed, and COVID-19 non-high risk. Robust standard errors in parentheses. ${ }^{* * *} \mathrm{p}<0.01,{ }^{* *} \mathrm{p}<0.05,{ }^{*} \mathrm{p}<0.1$

\subsubsection{Working population}

In Table 9 we shift our attention to the working population, we find very similar results with regards to ethnicity and gender: (a) among males, BAME individuals experience a higher increase (8.7 pp, 95\% CI: [3.1,14.2]) in mental health problems compared to British White individuals; (b) among females, BAME individuals experience a non-statistically significant lower increase $(-4.5 \mathrm{pp}, 95 \%$ CI: $[-9.9,1.0])$ in mental health problems compared to British White individuals; (c) among British White individuals, females experience a higher increase (10.5 pp, 95\% CI: $[7.6,13.4]$ ) in mental health problems compared to men; (d) among BAME individuals, females experience a non-statistically significant lower increase (-2.6 pp, 95\% CI: $[-9.6,4.3)$ in mental health problems compared to men. We also find that occupation matters: individuals categorised as working in "small employers \& own account" occupations have experienced a larger increase (6.8 pp) in mental health problems compared to individuals working in "managerial and professional" occupations. ${ }^{13}$ Table 10 shows once again substantial heterogeneity across BAME groups. ${ }^{14}$

\footnotetext{
13 Table A7 in Appendix C uses an indicator for White vs. non-White individuals.

${ }^{14}$ Table A8 in Appendix C uses an indicator for BIP vs. non-BIP individuals.
} 
Table 9: OLS regressions of percentage point changes in mental health problems in the UKHLS from Wave 9 (2017-2019) to April 2020 among working individuals (employed or self-employed) on BAME and other characteristics

(1)

$(2)$

(3)

(4)

(5)

\begin{tabular}{|c|c|c|c|c|c|}
\hline$<25$ & $\begin{array}{c}0.032 \\
(0.044)\end{array}$ & $\begin{array}{c}0.032 \\
(0.044)\end{array}$ & $\begin{array}{c}0.017 \\
(0.050)\end{array}$ & $\begin{array}{c}0.042 \\
(0.054)\end{array}$ & $\begin{array}{c}0.043 \\
(0.055)\end{array}$ \\
\hline \multirow[t]{2}{*}{$25-34$} & $0.089 * * *$ & $0.089 * * *$ & $0.072 * *$ & $0.076^{* *}$ & $0.077^{* *}$ \\
\hline & $(0.028)$ & $(0.028)$ & $(0.032)$ & $(0.036)$ & $(0.038)$ \\
\hline \multirow[t]{2}{*}{$35-44$} & 0.037 & 0.036 & 0.025 & 0.028 & 0.029 \\
\hline & $(0.026)$ & $(0.026)$ & $(0.029)$ & $(0.034)$ & $(0.035)$ \\
\hline \multirow[t]{2}{*}{$45-54$} & 0.015 & 0.014 & 0.005 & 0.010 & 0.012 \\
\hline & $(0.025)$ & $(0.025)$ & $(0.028)$ & $(0.032)$ & $(0.033)$ \\
\hline \multirow[t]{2}{*}{$55-64$} & 0.003 & 0.003 & 0.002 & -0.006 & -0.005 \\
\hline & $(0.025)$ & $(0.025)$ & $(0.028)$ & $(0.032)$ & $(0.032)$ \\
\hline \multirow[t]{2}{*}{ BAME } & 0.010 & $0.082^{* * *}$ & $0.081^{* * *}$ & $0.087^{* * *}$ & $0.087^{* * *}$ \\
\hline & $(0.017)$ & $(0.024)$ & $(0.027)$ & $(0.028)$ & $(0.028)$ \\
\hline \multirow[t]{2}{*}{ Female } & $0.083^{* * *}$ & $0.103^{* * *}$ & $0.099 * * *$ & $0.105^{* * *}$ & $0.105^{* * *}$ \\
\hline & $(0.012)$ & $(0.013)$ & $(0.014)$ & $(0.015)$ & $(0.015)$ \\
\hline \multirow[t]{2}{*}{ BAME $\times$ Female } & & $-0.126^{* * *}$ & $-0.139 * * *$ & $-0.131^{* * *}$ & $-0.131^{* * *}$ \\
\hline & & $(0.033)$ & $(0.036)$ & $(0.038)$ & $(0.038)$ \\
\hline \multirow[t]{2}{*}{ F2F interview baseline } & 0.011 & 0.011 & 0.011 & 0.008 & 0.008 \\
\hline & $(0.013)$ & $(0.013)$ & $(0.014)$ & $(0.015)$ & $(0.015)$ \\
\hline \multirow[t]{2}{*}{ Household size } & 0.005 & 0.005 & 0.006 & 0.004 & 0.004 \\
\hline & $(0.005)$ & $(0.005)$ & $(0.005)$ & $(0.005)$ & $(0.005)$ \\
\hline \multirow[t]{2}{*}{ Living with a partner } & & & 0.029 & 0.031 & 0.031 \\
\hline & & & $(0.053)$ & $(0.055)$ & $(0.055)$ \\
\hline \multirow[t]{2}{*}{ Divorced or separated } & & & 0.027 & 0.040 & 0.040 \\
\hline & & & $(0.056)$ & $(0.059)$ & $(0.059)$ \\
\hline \multirow[t]{2}{*}{ Never married } & & & 0.047 & 0.052 & 0.052 \\
\hline & & & $(0.056)$ & $(0.058)$ & $(0.059)$ \\
\hline \multirow[t]{2}{*}{ London } & & & 0.030 & 0.016 & 0.016 \\
\hline & & & $(0.023)$ & $(0.024)$ & $(0.024)$ \\
\hline \multirow[t]{2}{*}{ Wales } & & & 0.030 & 0.018 & 0.018 \\
\hline & & & $(0.029)$ & $(0.030)$ & $(0.030)$ \\
\hline \multirow[t]{2}{*}{ Scotland } & & & 0.037 & 0.031 & 0.031 \\
\hline & & & $(0.023)$ & $(0.025)$ & $(0.025)$ \\
\hline \multirow[t]{2}{*}{ Northern Ireland } & & & -0.001 & 0.016 & 0.016 \\
\hline & & & $(0.035)$ & $(0.037)$ & $(0.037)$ \\
\hline \multirow[t]{2}{*}{ BA or higher } & & & $0.037^{*}$ & 0.033 & 0.033 \\
\hline & & & $(0.019)$ & $(0.022)$ & $(0.022)$ \\
\hline \multirow[t]{2}{*}{ Diploma or equiv. } & & & 0.013 & 0.010 & 0.010 \\
\hline & & & $(0.025)$ & $(0.026)$ & $(0.026)$ \\
\hline \multirow[t]{2}{*}{ A Level or equiv. } & & & 0.004 & -0.008 & -0.007 \\
\hline & & & $(0.026)$ & $(0.028)$ & $(0.028)$ \\
\hline \multirow[t]{2}{*}{ GCSE or equiv. } & & & -0.000 & -0.001 & -0.001 \\
\hline & & & $(0.020)$ & $(0.022)$ & $(0.022)$ \\
\hline \multirow[t]{2}{*}{ Self-employed } & & & & -0.014 & -0.014 \\
\hline & & & & $(0.029)$ & $(0.029)$ \\
\hline \multirow[t]{2}{*}{ Net personal income } & & & & 0.002 & 0.002 \\
\hline & & & & $(0.003)$ & $(0.003)$ \\
\hline Intermediate & & & & -0.000 & -0.000 \\
\hline & & & & $(0.021)$ & $(0.021)$ \\
\hline
\end{tabular}


Small employers \& own account

$\begin{array}{cc}0.068^{* *} & 0.068^{* *} \\ (0.034) & (0.034) \\ 0.019 & 0.019 \\ (0.029) & (0.029) \\ -0.015 & -0.014 \\ (0.019) & (0.019) \\ & 0.002 \\ & (0.016)\end{array}$

\begin{tabular}{llllll}
\hline $\mathrm{N}$ & 8,695 & 8,695 & 7,729 & 7,022 & 7,021 \\
$\mathrm{R}$-squared & 0.010 & 0.012 & 0.013 & 0.014 & 0.014 \\
\hline
\end{tabular}

Note: All regressions include month of (baseline) interview fixed effects. The reference categories are: British White, male, aged 65 or older, online interview in 2017-2019, interview in April in 2017-2019, widowed, living in England (excluding London), no qualification, employed, Management and Professional, COVID-19 non-high risk. Robust standard errors in parentheses. ${ }^{* * *} \mathrm{p}<0.01,{ }^{* *} \mathrm{p}<0.05,{ }^{*} \mathrm{p}<0.1$

Table 10: OLS regressions of percentage point changes in mental health problems in the UKHLS from Wave 9 (2017-2019) to April 2020 (employed or self-employed) on BAME groups and other characteristics

(1)

\begin{tabular}{|c|c|c|c|c|c|}
\hline & (1) & $(2)$ & (3) & (4) & (5) \\
\hline \multirow[t]{2}{*}{$<25$} & 0.030 & 0.031 & 0.012 & 0.036 & 0.037 \\
\hline & $(0.044)$ & $(0.044)$ & $(0.050)$ & $(0.054)$ & $(0.055)$ \\
\hline \multirow[t]{2}{*}{$25-34$} & $0.089 * * *$ & $0.089 * * *$ & $0.070^{* *}$ & $0.074^{* *}$ & $0.075^{* *}$ \\
\hline & $(0.028)$ & $(0.028)$ & $(0.032)$ & $(0.036)$ & $(0.038)$ \\
\hline \multirow{2}{*}{$35-44$} & 0.038 & 0.036 & 0.023 & 0.026 & 0.027 \\
\hline & $(0.026)$ & $(0.026)$ & $(0.029)$ & $(0.034)$ & $(0.035)$ \\
\hline \multirow[t]{2}{*}{$45-54$} & 0.017 & 0.015 & 0.006 & 0.011 & 0.012 \\
\hline & $(0.025)$ & $(0.025)$ & $(0.028)$ & $(0.032)$ & $(0.033)$ \\
\hline \multirow[t]{2}{*}{$55-64$} & 0.004 & 0.003 & 0.002 & -0.005 & -0.004 \\
\hline & $(0.025)$ & $(0.025)$ & $(0.028)$ & $(0.032)$ & $(0.032)$ \\
\hline \multirow[t]{2}{*}{ Other White } & 0.014 & 0.066 & $0.087^{*}$ & 0.081 & 0.081 \\
\hline & $(0.028)$ & $(0.043)$ & $(0.052)$ & $(0.053)$ & $(0.053)$ \\
\hline \multirow[t]{2}{*}{ Mixed } & -0.066 & -0.032 & -0.049 & -0.043 & -0.043 \\
\hline & $(0.051)$ & $(0.072)$ & $(0.075)$ & $(0.080)$ & $(0.080)$ \\
\hline \multirow[t]{2}{*}{ Black } & -0.044 & 0.046 & 0.034 & 0.035 & 0.035 \\
\hline & $(0.038)$ & $(0.056)$ & $(0.061)$ & $(0.064)$ & $(0.064)$ \\
\hline \multirow[t]{2}{*}{ BIP } & $0.079 * * *$ & $0.170^{* * *}$ & $0.157^{* * *}$ & $0.174^{* * *}$ & $0.174^{* * *}$ \\
\hline & $(0.028)$ & $(0.036)$ & $(0.038)$ & $(0.040)$ & $(0.040)$ \\
\hline \multirow[t]{2}{*}{ Chinese } & -0.088 & -0.058 & -0.081 & -0.076 & -0.076 \\
\hline & $(0.057)$ & $(0.074)$ & $(0.080)$ & $(0.082)$ & $(0.082)$ \\
\hline \multirow[t]{2}{*}{ Arab } & 0.175 & 0.170 & 0.209 & 0.213 & 0.214 \\
\hline & $(0.145)$ & $(0.186)$ & $(0.153)$ & $(0.155)$ & $(0.155)$ \\
\hline \multirow[t]{2}{*}{ Female } & $0.085^{* * *}$ & $0.103^{* * *}$ & $0.100^{* * *}$ & $0.104^{* * *}$ & $0.104^{* * *}$ \\
\hline & $(0.012)$ & $(0.013)$ & $(0.014)$ & $(0.015)$ & $(0.015)$ \\
\hline \multirow[t]{2}{*}{ Other White $\times$ Female } & & -0.085 & $-0.127^{*}$ & -0.098 & -0.099 \\
\hline & & $(0.056)$ & $(0.065)$ & $(0.068)$ & $(0.068)$ \\
\hline \multirow[t]{2}{*}{ Mixed $\times$ Female } & & -0.060 & -0.052 & -0.068 & -0.068 \\
\hline & & $(0.100)$ & $(0.104)$ & $(0.109)$ & $(0.109)$ \\
\hline \multirow[t]{2}{*}{ Black $\times$ Female } & & $-0.141^{*}$ & $-0.153^{*}$ & $-0.147^{*}$ & $-0.146^{*}$ \\
\hline & & $(0.075)$ & $(0.078)$ & $(0.083)$ & $(0.083)$ \\
\hline \multirow[t]{2}{*}{ BIP $\times$ Female } & & $-0.182^{* * *}$ & $-0.179 * * *$ & $-0.179 * * *$ & $-0.179 * * *$ \\
\hline & & $(0.055)$ & $(0.057)$ & $(0.061)$ & $(0.061)$ \\
\hline
\end{tabular}




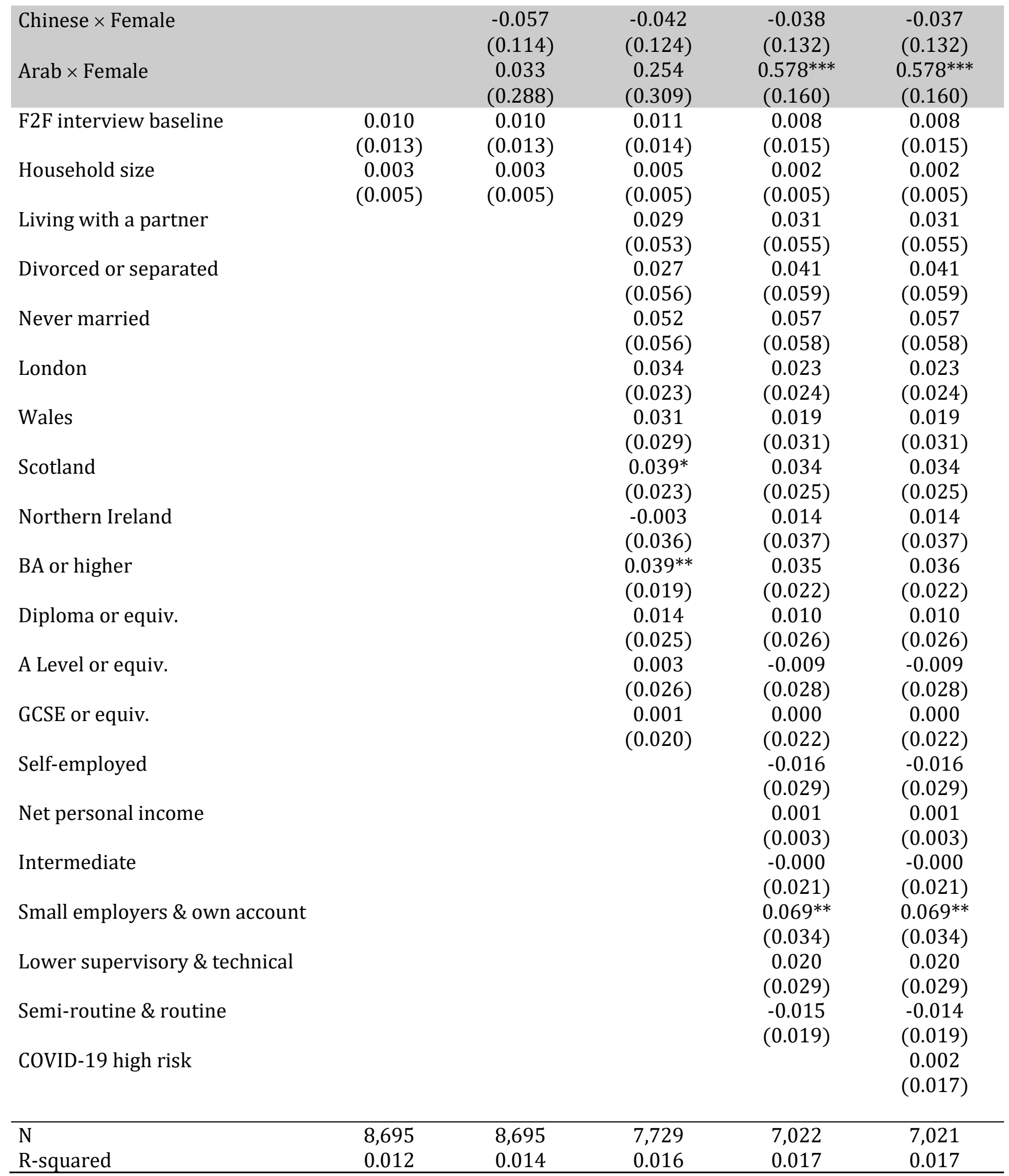

Note: All regressions include month of (baseline) interview fixed effects. The reference categories are: British White, male, aged 65 or older, online interview in 2017-2019, interview in April in 2017-2019, widowed, living in England (excluding London), no qualification, employed, Management and Professional, COVID-19 non-high risk. Robust standard errors in parentheses. ${ }^{* * *} \mathrm{p}<0.01,{ }^{* *} \mathrm{p}<0.05,{ }^{*} \mathrm{p}<0.1$ 


\section{Discussion}

This study complements and extends previous research on the heterogeneous effects of COVID-19 and its pandemic across BAME groups. We find evidence that with the COVID-19 pandemic the mental health of BAME male individuals has deteriorated much more than that of British White individuals. Among women, both BAME and British White individuals have experienced similar drops in mental health. A by-product of these two findings is that the gender gap in mental health has increased among British White individuals, but not among BAME individuals.

We also find that the BAME differences seem to be driven by Bangladeshi, Indian and Pakistani individuals. The differential drop in mental health between Arab and British White is also quite large, albeit imprecisely estimated on account of the very small number of individuals in this BAME group.

The documented magnitudes are substantial: among males, the mental health of BAME individuals has deteriorated 0.13-0.14 standard deviations more than that of British White individuals. Future work should try to investigate the reasons and mechanisms behind the documented differential drops in mental health by ethnicity and gender.

We envisage two different, albeit complementary, approaches. The first consists in exploiting additional information available in the UKHLS. One may for instance use the key worker or NHS variables to take into account the unbalance in the share of key workers among minority ethnic groups with respect to British White individuals (e.g., "Indian men are 150\% more likely to work in health or social care roles than their white British counterparts", Platt and Warwick, 2020). The second approach is based on the collection of new data, and it can be either exploratory or confirmatory work. In former case there is no need to pre-register the hypothesis to be tested. In the latter case pre-registration can add credibility to the investigation of the mechanisms being tested. 


\section{References}

Adams-Prassl, A., Boneva, T., Golin M., \& Rauh, C. (2020). "The Impact of the Coronavirus Lockdown on Mental Health: Evidence from the US." Working Paper 2020-030, Human Capital and Economic Opportunity Working Group.

Aalto, A. M., Elovainio, M., Kivimaki, M., Uutela, A., \& Pirkola, S. (2012). “The beck depression inventory and general health questionnaire as measures of depression in the general population: a validation study using the composite international diagnostic interview as the gold standard." Psychiatry Research, 197 (1-2):163-171.

Banks, J. \& Xu, X. (2020). "The mental health effects of the first two months of lockdown and social distancing during the Covid-19 pandemic in the UK." Covid Economics 28, 12 June 2020: 91-118. CEPR press.

Daly, M., Sutin, A., Robinson, E. (2020). “Longitudinal changes in mental health and the COVID-19 pandemic: Evidence from the UK Household Longitudinal Study." https://doi.org/10.31234/osf.io/qd5z7

Davillas, A. \& Jones, A. M. (2020). "The COVID-19 pandemic and its impact on inequality of opportunity in psychological distress in the UK." ISER Working Paper Series 2020-07.

Dolan, P. \& Kavetsos, G. (2016). "Happy Talk: Mode of Administration Effects on Subjective Well-Being." Journal of Happiness Studies, 17:1273-1291.

Etheridge, B. \& Spanting, L. (2020). "The gender gap in mental well-being during the Covid-19 outbreak: Evidence from the UK." Covid Economics 33, 30 June 2020: 46-72. CEPR press.

Goldberg, D., \& Williams, P. (1988). “A User's Guide to the General health Questionnaire." Windsor: NFER-Nelson.

Goldberg, D., Gater, R., Sartorius, N., Üstün, T., Piccinelli, M., Gureje, O., Rutter, C. (1997). "The validity of two versions of the GHQ in the WHO study of mental illness in general health care." Psychological Medicine 27:191-7.

Kirby, T. (2020). "Evidence mounts on the disproportionate effect of COVID19 on ethnic minorities." The Lancet Respiratory Medicine, 8(6), 547-548.

Oreffice, S. \& Quintana-Domeque, C. (2020). “Gender inequality in COVID-19 times: Evidence from UK Prolific participants.” IZA Discussion Paper 13463.

Platt, L. \& Warwick, R. (2020). "Are some ethnic groups more vulnerable to COVID-19 than others?" The Deaton Review, Institute for Fiscal Studies. 
Proto, E. (2016). “Are happy workers more productive?” IZA World of Labor 2016: 315.

Public Health England (2020a). Disparities in the risk and outcomes of COVID-19: https://www.gov.uk/government/publications/covid-19-review-ofdisparities-in-risks-and-outcomes

Public Health England (2020b). Beyond the data: Understanding the impact of COVID-19 on groups: https://www.gov.uk/government/publications/covid-19-understanding-theimpact-on-bame-communities

Razaq, A., Harrison, D., Barr, B., Asaria, M., Routen, A., \& Khunti, K. (2020). BAME Covid-19 Deaths-what do we know? Rapid data \& evidence review. Oxford COVID-19 Evidence Service: https://www.cebm.net/covid-19/bame-covid-19deaths-what-do-we-know-rapid-data-evidence-review 


\section{APPENDIX}

Appendix A. Variables used in the analysis.

\section{Outcome (dependent) variables:}

- The standardised GHQ is the subjective wellbeing (GHQ): Likert (scghq1_dv) minus its sample mean divided by its standard deviation. scghq1_dv "converts valid answers to 12 questions of the General Health Questionnaire (GHQ) to a single scale by recoding so that the scale for individual variables runs from 0 to 3 instead of 1 to 4 , and then summing, giving a scale running from 0 (the least distressed) to 36 (the most distressed)."15

- The mental health problems indicator equals 1 if the subjective wellbeing (GHQ): Caseness (scghq2_dv) is 3 or above, and 0 otherwise. scghq2_dv "converts valid answers to 12 questions of the General Health Questionnaire (GHQ) to a single scale by recoding 1 and 2 values on individual variables to 0 , and 3 and 4 values to 1 , and then summing, giving a scale running from 0 (the least distressed) to 12 (the most distressed)."

\section{Explanatory (independent) variables:}

- The age group indicators are created using age_dv. "age_dv is the sample member's age at time of the interview derived from date of birth held in the sample administration data base and the derived date of interview. Recoded to missing for sample members whose interview outcome is inconsistent with the suggested age +/- one year."

- The ethnic group indicators are created using racel_dv, which "uses information collected in interviews with adults and includes information collected in previous interviews and from other members of the household."

- Gender (or sex) indicator is based on sex_dv, the respondent's sex, derived and cross-wave checked, which "assumes a value of 1 if all information in the study suggests the respondent is female, 2 if male and 0 if the information is inconsistent and the forename listed in the survey administration data base also does not suggest a particular gender."

- The face-to-face interview indicator is based on indmode.

- Household size is based on the sum of the household composition indicators: hhcompa (Household composition - Aged 0-4), hhcompb (Household composition - Aged 5-15), hhcompc (Household composition Aged 16-18), hhcompd (Household composition - Aged 19-69) and hhcompe (Household composition - Aged 70 or older).

15 This description and all that follow on this page in "' " are borrowed from: https://www.understandingsociety.ac.uk/documentation/mainstage/dataset-documentation/variable Additional information can be found following the link and references therein. 
- The marital status indicators are created using de facto marital status (marstat_dv).

- The location indicators are created using Government Office Region (gor_dv).

- The educational group indicators are created using the current status highest educational qualification (qfhigh_dv). "The information is updated each year to include the most recent qualifications of new entrants and existing panel members. The last highest educational qualification derived variable is fed forward and then updated with new qualifications obtained."

- The employment status is based on the respondent employment status at previous interview (ff_jbstat).

- The income measure is the monthly total net personal income - no deductions (fimnnet_dv) divided by 1,000. fimnnet_dv is "top-coded at +/$£ 15,000$ (as applicable) in the standard end-user licence data (UKDS SN6614). No top-coding applied in Special Licence data (UKDS SN6931)."

- The type of job indicators are based on current job (jbnssec5_dv): "five categories version of the National Statistics Socio-economic Classification (NS-SEC)."

- The COVID-19 high risk indicator used in our study is 1 if either the COVID19 high risk (clinically vulnerable) indicator (hrisk_dv) or the COVID-19 very high risk (clinically extremely vulnerable) (vhrisk_dv) is 1 , and 0 otherwise.

- The month of interview indicators are based on date of interview, month (intdatm_dv). 


\section{Appendix B. GHQ-12 Questionnaire.}

\section{The GHQ module contains the following questions for the GHQ-12 Questionnaire:}

- $\quad$ scghqa [GHQ: concentration] The next questions are about how you have been feeling over the last few weeks. Have you recently been able to concentrate on whatever you're doing?

1. Better than usual 2. Same as usual 3. Less than usual 4. Much less than usual

- $\quad$ scghqb [GHQ: loss of sleep] Have you recently lost much sleep over worry?

1. Not at all 2. No more than usual 3. Rather more than usual 4. Much more than usual

- $\quad$ scghqc [GHQ: playing a useful role] Have you recently felt that you were playing a useful part in things?

1. More so than usual 2. Same as usual 3. Less so than usual 4. Much less than usual

- $\quad$ scghqd [GHQ: capable of making decisions] Have you recently felt capable of making decisions about things?

1. More so than usual 2. Same as usual 3. Less so than usual 4. Much less capable

- $\quad$ scghqe [GHQ: constantly under strain] Have you recently felt constantly under strain?

1. Not at all 2. No more than usual 3. Rather more than usual 4. Much more than usual

- $\quad$ scghqf [GHQ: problem overcoming difficulties] Have you recently felt you couldn't overcome your difficulties?

1. Not at all 2. No more than usual 3. Rather more than usual 4. Much more than usual

- $\quad$ scghqg [GHQ: enjoy day-to-day activities] Have you recently been able to enjoy your normal day-today activities?

1. More so than usual 2. Same as usual 3. Less so than usual 4. Much less than usual

- $\quad$ scghqh [GHQ: ability to face problems] Have you recently been able to face up to problems?

1. More so than usual 2. Same as usual 3. Less able than usual 4. Much less able

- $\quad$ scghqi [GHQ: unhappy or depressed] Have you recently been feeling unhappy or depressed?

1. Not at all 2. No more than usual 3. Rather more than usual 4. Much more than usual

- $\quad$ scghqj [GHQ: losing confidence] Have you recently been losing confidence in yourself?

1. Not at all 2. No more than usual 3. Rather more than usual 4. Much more than usual

- scghqk [GHQ: believe worthless] Have you recently been thinking of yourself as a worthless person?

1. Not at all 2. No more than usual 3. Rather more than usual 4. Much more than usual 
- scghql [GHQ: general happiness] Have you recently been feeling reasonably happy, all things considered?

1. More so than usual 2. About the same as usual 3. Less so than usual 4. Much less than usual

The GHQ-12 range goes from $0-36$. This range is obtained by subtracting 1 to the values given in each question. Thus, the values in each question are re-coded from 1-4 to $0-3$. 
Appendix C. Additional Regressions.

Table A1: OLS regressions of (standardised) changes in mental distress in the UKHLS from Wave 9 (20172019) to April 2020 on White and other characteristics

\begin{tabular}{|c|c|c|c|c|c|}
\hline$<25$ & $\begin{array}{c}0.117^{* * *} \\
(0.043)\end{array}$ & $\begin{array}{c}0.119^{* * *} \\
(0.043)\end{array}$ & $\begin{array}{c}0.061 \\
(0.056)\end{array}$ & $\begin{array}{c}0.104 \\
(0.074)\end{array}$ & $\begin{array}{c}0.092 \\
(0.076)\end{array}$ \\
\hline $25-34$ & $\begin{array}{c}0.105^{* * *} \\
(0.032)\end{array}$ & $\begin{array}{c}0.107^{* * *} \\
(0.032)\end{array}$ & $\begin{array}{l}0.072 * * \\
(0.036)\end{array}$ & $\begin{array}{c}0.151^{* * *} \\
(0.046)\end{array}$ & $\begin{array}{c}0.140^{* * *} \\
(0.049)\end{array}$ \\
\hline $35-44$ & $\begin{array}{c}0.001 \\
(0.030)\end{array}$ & $\begin{array}{c}0.001 \\
(0.030)\end{array}$ & $\begin{array}{c}-0.014 \\
(0.033)\end{array}$ & $\begin{array}{c}0.075^{*} \\
(0.042)\end{array}$ & $\begin{array}{c}0.064 \\
(0.045)\end{array}$ \\
\hline $45-54$ & $\begin{array}{c}-0.116^{* * *} \\
(0.025)\end{array}$ & $\begin{array}{c}-0.118^{* * *} \\
(0.025)\end{array}$ & $\begin{array}{c}-0.138^{* * * *} \\
(0.028)\end{array}$ & $\begin{array}{c}-0.052 \\
(0.038)\end{array}$ & $\begin{array}{c}-0.061 \\
(0.040)\end{array}$ \\
\hline $55-64$ & $\begin{array}{c}-0.055^{* *} \\
(0.022)\end{array}$ & $\begin{array}{c}-0.055^{* *} \\
(0.022)\end{array}$ & $\begin{array}{c}-0.060^{* *} \\
(0.024)\end{array}$ & $\begin{array}{c}0.011 \\
(0.031)\end{array}$ & $\begin{array}{c}0.003 \\
(0.033)\end{array}$ \\
\hline White & $\begin{array}{c}-0.018 \\
(0.032)\end{array}$ & $\begin{array}{c}-0.140^{* * *} \\
(0.044)\end{array}$ & $\begin{array}{c}-0.102^{* *} \\
(0.047)\end{array}$ & $\begin{array}{c}-0.126^{* * *} \\
(0.048)\end{array}$ & $\begin{array}{c}-0.127^{* * *} \\
(0.048)\end{array}$ \\
\hline Female & $\begin{array}{c}0.124^{* * *} \\
(0.017)\end{array}$ & $\begin{array}{l}-0.062 \\
(0.058)\end{array}$ & $\begin{array}{c}-0.054 \\
(0.060)\end{array}$ & $\begin{array}{l}-0.068 \\
(0.063)\end{array}$ & $\begin{array}{c}-0.069 \\
(0.063)\end{array}$ \\
\hline White $\times$ Female & & $\begin{array}{c}0.205^{* * *} \\
(0.061)\end{array}$ & $\begin{array}{c}0.204^{* * *} \\
(0.063)\end{array}$ & $\begin{array}{c}0.223^{* * *} \\
(0.065)\end{array}$ & $\begin{array}{c}0.223^{* * *} \\
(0.065)\end{array}$ \\
\hline F2F interview baseline & $\begin{array}{l}0.038^{* *} \\
(0.019)\end{array}$ & $\begin{array}{l}0.038^{* *} \\
(0.019)\end{array}$ & $\begin{array}{l}0.045^{* *} \\
(0.020)\end{array}$ & $\begin{array}{l}0.047^{* *} \\
(0.021)\end{array}$ & $\begin{array}{l}0.047^{* *} \\
(0.021)\end{array}$ \\
\hline Household size & $\begin{array}{c}0.010 \\
(0.008)\end{array}$ & $\begin{array}{c}0.010 \\
(0.008)\end{array}$ & $\begin{array}{c}0.013 \\
(0.009)\end{array}$ & $\begin{array}{c}0.014 \\
(0.009)\end{array}$ & $\begin{array}{c}0.014 \\
(0.009)\end{array}$ \\
\hline Living with a partner & & & $\begin{array}{c}-0.013 \\
(0.044)\end{array}$ & $\begin{array}{c}-0.024 \\
(0.045)\end{array}$ & $\begin{array}{l}-0.026 \\
(0.045)\end{array}$ \\
\hline Divorced or separated & & & $\begin{array}{c}-0.046 \\
(0.055)\end{array}$ & $\begin{array}{c}-0.039 \\
(0.055)\end{array}$ & $\begin{array}{c}-0.040 \\
(0.055)\end{array}$ \\
\hline Never married & & & $\begin{array}{c}0.041 \\
(0.054)\end{array}$ & $\begin{array}{c}0.048 \\
(0.055)\end{array}$ & $\begin{array}{c}0.046 \\
(0.055)\end{array}$ \\
\hline London & & & $\begin{array}{c}0.090^{* * *} \\
(0.033)\end{array}$ & $\begin{array}{c}0.094^{* * *} \\
(0.034)\end{array}$ & $\begin{array}{c}0.094^{* * *} \\
(0.034)\end{array}$ \\
\hline Wales & & & $\begin{array}{c}0.043 \\
(0.042)\end{array}$ & $\begin{array}{c}0.063 \\
(0.041)\end{array}$ & $\begin{array}{c}0.063 \\
(0.042)\end{array}$ \\
\hline Scotland & & & $\begin{array}{c}0.090^{* * *} \\
(0.034)\end{array}$ & $\begin{array}{c}0.091^{* * *} \\
(0.035)\end{array}$ & $\begin{array}{c}0.091^{* * *} \\
(0.035)\end{array}$ \\
\hline Northern Ireland & & & $\begin{array}{c}-0.006 \\
(0.051)\end{array}$ & $\begin{array}{l}-0.005 \\
(0.052)\end{array}$ & $\begin{array}{c}-0.005 \\
(0.052)\end{array}$ \\
\hline BA or higher & & & $\begin{array}{c}0.073^{* * *} \\
(0.027)\end{array}$ & $\begin{array}{c}0.042 \\
(0.028)\end{array}$ & $\begin{array}{c}0.041 \\
(0.028)\end{array}$ \\
\hline Diploma or equiv. & & & $\begin{array}{c}0.029 \\
(0.034)\end{array}$ & $\begin{array}{c}0.016 \\
(0.034)\end{array}$ & $\begin{array}{c}0.016 \\
(0.034)\end{array}$ \\
\hline A Level or equiv. & & & $\begin{array}{c}0.031 \\
(0.036)\end{array}$ & $\begin{array}{c}0.010 \\
(0.037)\end{array}$ & $\begin{array}{c}0.009 \\
(0.037)\end{array}$ \\
\hline GCSE or equiv. & & & $\begin{array}{c}0.011 \\
(0.028)\end{array}$ & $\begin{array}{c}0.003 \\
(0.028)\end{array}$ & $\begin{array}{c}0.002 \\
(0.028)\end{array}$ \\
\hline Self-employed & & & & $\begin{array}{l}0.085^{* *} \\
(0.036)\end{array}$ & $\begin{array}{l}0.085^{* *} \\
(0.036)\end{array}$ \\
\hline
\end{tabular}


Unemployed

Retired

House-keeper

Student

Disabled

Other

Net personal income

COVID-19 high risk

$\begin{array}{cc}-0.117^{*} & -0.116^{*} \\ (0.069) & (0.069) \\ 0.093^{* * *} & 0.094^{* * *} \\ (0.032) & (0.032) \\ -0.017 & -0.017 \\ (0.056) & (0.056) \\ 0.074 & 0.074 \\ (0.074) & (0.074) \\ -0.367^{* * *} & -0.362^{* * *} \\ (0.079) & (0.079) \\ 0.146 & 0.146 \\ (0.114) & (0.114) \\ 0.003 & 0.003 \\ (0.005) & (0.005) \\ & -0.017 \\ & (0.024)\end{array}$

$\mathrm{N}$
$\mathrm{R}$-squared
14,155

0.012
14,155

0.012
12,000

0.021
11,999

0.021 
Table A2: OLS regressions of (standardised) changes in mental distress in the UKHLS from Wave 9 (2017-2019) to April 2020 on BIP and other characteristics

(1)

\begin{tabular}{|c|c|c|c|c|c|}
\hline$<25$ & $\begin{array}{c}0.112^{* * *} \\
(0.043)\end{array}$ & $\begin{array}{c}0.114^{* * *} \\
(0.043)\end{array}$ & $\begin{array}{c}0.058 \\
(0.056)\end{array}$ & $\begin{array}{c}0.100 \\
(0.074)\end{array}$ & $\begin{array}{c}0.086 \\
(0.076)\end{array}$ \\
\hline \multirow[t]{2}{*}{$25-34$} & $0.103^{* * *}$ & $0.105^{* * *}$ & $0.069 *$ & $0.147^{* * *}$ & $0.134^{* * *}$ \\
\hline & $(0.032)$ & $(0.032)$ & $(0.036)$ & $(0.046)$ & $(0.049)$ \\
\hline \multirow[t]{2}{*}{$35-44$} & 0.001 & 0.000 & -0.016 & $0.071^{*}$ & 0.059 \\
\hline & $(0.030)$ & $(0.030)$ & $(0.033)$ & $(0.042)$ & $(0.045)$ \\
\hline \multirow[t]{2}{*}{$45-54$} & $-0.115^{* * *}$ & $-0.117^{* * *}$ & $-0.138^{* * *}$ & -0.053 & -0.064 \\
\hline & $(0.025)$ & $(0.025)$ & $(0.028)$ & $(0.038)$ & $(0.040)$ \\
\hline \multirow[t]{2}{*}{$55-64$} & $-0.054^{* *}$ & $-0.054^{* *}$ & $-0.061^{* *}$ & 0.009 & 0.001 \\
\hline & $(0.022)$ & $(0.022)$ & $(0.024)$ & $(0.031)$ & $(0.033)$ \\
\hline \multirow[t]{2}{*}{ BIP } & $0.109 * *$ & $0.261^{* * *}$ & $0.228^{* * *}$ & $0.260^{* * *}$ & $0.261^{* * *}$ \\
\hline & $(0.044)$ & $(0.059)$ & $(0.059)$ & $(0.062)$ & $(0.062)$ \\
\hline \multirow[t]{2}{*}{ Female } & $0.125^{* * *}$ & $0.138^{* * *}$ & $0.144^{* * *}$ & $0.148^{* * *}$ & $0.147^{* * *}$ \\
\hline & $(0.017)$ & $(0.017)$ & $(0.018)$ & $(0.019)$ & $(0.019)$ \\
\hline \multirow[t]{2}{*}{ BIP $\times$ Female } & & $-0.266^{* * *}$ & $-0.259 * * *$ & $-0.273^{* * *}$ & $-0.273^{* * *}$ \\
\hline & & $(0.084)$ & $(0.085)$ & $(0.090)$ & $(0.090)$ \\
\hline \multirow[t]{2}{*}{ F2F interview baseline } & $0.037^{*}$ & $0.037^{*}$ & $0.043^{* *}$ & $0.045^{* *}$ & $0.046^{* *}$ \\
\hline & $(0.019)$ & $(0.019)$ & $(0.020)$ & $(0.021)$ & $(0.021)$ \\
\hline \multirow{2}{*}{ Household size } & 0.007 & 0.007 & 0.010 & 0.011 & 0.011 \\
\hline & $(0.008)$ & $(0.008)$ & $(0.009)$ & $(0.009)$ & $(0.009)$ \\
\hline \multirow[t]{2}{*}{ Living with a partner } & & & -0.012 & -0.024 & -0.026 \\
\hline & & & $(0.044)$ & $(0.045)$ & $(0.045)$ \\
\hline \multirow[t]{2}{*}{ Divorced or separated } & & & -0.048 & -0.041 & -0.043 \\
\hline & & & $(0.054)$ & $(0.055)$ & $(0.055)$ \\
\hline \multirow[t]{2}{*}{ Never married } & & & 0.039 & 0.046 & 0.044 \\
\hline & & & $(0.054)$ & $(0.055)$ & $(0.055)$ \\
\hline \multirow[t]{2}{*}{ London } & & & $0.074^{* *}$ & $0.079 * *$ & $0.079 * *$ \\
\hline & & & $(0.032)$ & $(0.033)$ & $(0.033)$ \\
\hline \multirow[t]{2}{*}{ Wales } & & & 0.048 & 0.067 & 0.067 \\
\hline & & & $(0.042)$ & $(0.042)$ & $(0.042)$ \\
\hline \multirow[t]{2}{*}{ Scotland } & & & $0.095^{* * *}$ & $0.096^{* * *}$ & $0.096^{* * *}$ \\
\hline & & & $(0.034)$ & $(0.035)$ & $(0.035)$ \\
\hline \multirow[t]{2}{*}{ Northern Ireland } & & & -0.000 & 0.001 & 0.001 \\
\hline & & & $(0.051)$ & $(0.052)$ & $(0.052)$ \\
\hline \multirow[t]{2}{*}{ BA or higher } & & & $0.071^{* * *}$ & 0.040 & 0.039 \\
\hline & & & $(0.027)$ & $(0.028)$ & $(0.028)$ \\
\hline \multirow[t]{2}{*}{ Diploma or equiv. } & & & 0.028 & 0.015 & 0.015 \\
\hline & & & $(0.034)$ & $(0.034)$ & $(0.034)$ \\
\hline \multirow[t]{2}{*}{ A Level or equiv. } & & & 0.029 & 0.007 & 0.007 \\
\hline & & & $(0.036)$ & $(0.037)$ & $(0.037)$ \\
\hline \multirow[t]{2}{*}{ GCSE or equiv. } & & & 0.010 & 0.002 & 0.002 \\
\hline & & & $(0.028)$ & $(0.028)$ & $(0.028)$ \\
\hline \multirow[t]{2}{*}{ Self-employed } & & & & $0.083^{* *}$ & $0.083^{* *}$ \\
\hline & & & & $(0.036)$ & $(0.036)$ \\
\hline Unemployed & & & & $-0.119 *$ & $-0.117^{*}$ \\
\hline & & & & $(0.069)$ & $(0.069)$ \\
\hline Retired & & & & $0.091^{* * *}$ & $0.092^{* * *}$ \\
\hline & & & & $(0.032)$ & $(0.032)$ \\
\hline House-keeper & & & & -0.026 & -0.025 \\
\hline
\end{tabular}


Student

(0.056) (0.056)

$0.070 \quad 0.071$

Disabled

(0.074) (0.074)

$-0.371^{* * *} \quad-0.365^{* * *}$

(0.078) (0.079)

Other

0.148

0.148

$(0.115)$

(0.115)

Net personal income

0.003

0.003

(0.005)

(0.005)

$-0.019$

COVID-19 high risk

(0.024)

\begin{tabular}{lccccc}
\hline $\mathrm{N}$ & 14,155 & 14,155 & 12,380 & 12,000 & 11,999 \\
$\mathrm{R}-$-squared & 0.012 & 0.013 & 0.016 & 0.021 & 0.021 \\
\hline
\end{tabular}


Table A3: OLS regressions of (standardised) changes in mental distress in the UKHLS from Wave 9 (20172019) to April 2020 among working individuals (employed or self-employed) on White and other characteristics

(1)

$<25$

25-34

35-44

45-54

$55-64$

White

Female

White $\times$ Female

F2F interview baseline

Household size

Living with a partner

Divorced or separated

Never married

London

Wales

Scotland

Northern Ireland

BA or higher

Diploma or equiv.

A Level or equiv.

GCSE or equiv.

Self-employed

Net personal income

Intermediate
(2)

(3)

(4)

(5)

$0.149 *$

0.151*

(0.081)

$0.151^{* * *}$

(0.081)

(0.048)

0.042

(0.046)

$-0.067$

(0.043)

$-0.003$

(0.042)

$-0.068^{*}$

(0.040)

$0.132^{* * *}$

(0.022)

$0.151^{* * *}$

(0.048)

0.040

(0.046)

$-0.069$

(0.043)

$-0.004$

(0.042)

$-0.205^{* *}$

(0.052)

$-0.094$

(0.074)

$0.251^{* * *}$

(0.077)

$0.060^{* *}$

(0.024)

$0.018^{*}$

(0.009)

$0.059 * *$

(0.024)

$0.017^{*}$

(0.009)

0.104

(0.092)

$0.116^{* *}$

(0.055)

0.027

(0.051)

$-0.091^{*}$

(0.048)

$-0.019$

(0.046)

$-0.182^{* * *}$

(0.055)

-0.088
$(0.076)$

(0.076)

$0.252^{* * *}$

(0.080)

$0.057^{* *}$

(0.026)

$0.021^{* *}$

(0.010)

0.046

(0.087)

$-0.005$

(0.095)

0.111

(0.094)

$0.075^{*}$

(0.042)

0.081

(0.051)

0.084*

(0.043)

$-0.042$

(0.065)

$-0.009$

(0.037)

$-0.053$

(0.047)

$-0.065$

(0.049)

$-0.050$

(0.039)

政

0.183
$(0.099)$
$0.170^{*}$

0.179*

$0.170^{* * * *}$

(0.061)

0.088

(0.057)

0.084

$-0.032$

(0.053)

$-0.036$

0.008

(0.052)

0.005

$-0.198^{* * *}$

(0.057)

$-0.067$

(0.081)

$-0.067$

$0.238^{* * *}$

(0.085)

$0.058^{* *}$

(0.027)

0.014

$0.059^{* *}$

(0.011)

0.014

$-0.015$

(0.011)

(0.091)

$-0.016$

$-0.032$

$-0.033$

(0.099)

0.044

0.044

(0.099)

0.042

0.042

(0.044)

0.047

0.047

(0.054)

0.069

0.069

(0.045)

0.015

0.015

(0.062)

$-0.001$

$-0.001$

(0.041)

$-0.040$

$-0.040$

(0.049)

$-0.075$

$-0.075$

(0.052)

$-0.053$

$-0.053$

(0.040)

(0.101)

$0.166^{* * *}$

(0.064)

(0.060)

(0.055)

(0.053)

$-0.199 * * *$

(0.057)

(0.081)

$0.238^{* * *}$

(0.085)

(0.027)

(0.091)

(0.099)

(0.099)

(0.044)

(0.054)

(0.045)

(0.062)

(0.041)

(0.049)

(0.052)

(0.040)

$-0.005$

$-0.004$

(0.057)

(0.057)

0.003

0.003

(0.006)

(0.006)

$-0.024$

$-0.024$

(0.038)

(0.038) 
Small employers \& own account

Lower supervisory \& technical

Semi-routine \& routine

COVID-19 high risk

$\begin{array}{cc}0.188^{* * *} & 0.188^{* * *} \\ (0.065) & (0.065) \\ 0.057 & 0.057 \\ (0.051) & (0.051) \\ 0.011 & 0.011 \\ (0.035) & (0.035) \\ & -0.007 \\ & (0.030)\end{array}$

8,695

8,695

0.014
7,729

0.019
7,022

0.021
7,021

0.021 
Table A4: OLS regressions of (standardised) changes in mental distress in the UKHLS from Wave 9 (2017-2019) to April 2020 among working individuals (employed or self-employed) on BIP and other characteristics

(1)

\begin{tabular}{|c|c|c|c|c|c|}
\hline & (1) & $(2)$ & (3) & (4) & (5) \\
\hline$<25$ & $\begin{array}{c}0.143^{*} \\
(0.081)\end{array}$ & $\begin{array}{c}0.146^{*} \\
(0.081)\end{array}$ & $\begin{array}{c}0.099 \\
(0.092)\end{array}$ & $\begin{array}{c}0.175^{*} \\
(0.099)\end{array}$ & $\begin{array}{c}0.169 * \\
(0.101)\end{array}$ \\
\hline $25-34$ & $\begin{array}{c}0.148^{* * *} \\
(0.048)\end{array}$ & $\begin{array}{c}0.148^{* * *} \\
(0.048)\end{array}$ & $\begin{array}{l}0.114^{* *} \\
(0.055)\end{array}$ & $\begin{array}{c}0.168^{* * *} \\
(0.061)\end{array}$ & $\begin{array}{l}0.162^{* *} \\
(0.064)\end{array}$ \\
\hline $35-44$ & $\begin{array}{c}0.042 \\
(0.046)\end{array}$ & $\begin{array}{c}0.039 \\
(0.046)\end{array}$ & $\begin{array}{c}0.025 \\
(0.051)\end{array}$ & $\begin{array}{c}0.085 \\
(0.057)\end{array}$ & $\begin{array}{c}0.080 \\
(0.060)\end{array}$ \\
\hline $45-54$ & $\begin{array}{c}-0.066 \\
(0.043)\end{array}$ & $\begin{array}{c}-0.068 \\
(0.043)\end{array}$ & $\begin{array}{c}-0.092^{*} \\
(0.048)\end{array}$ & $\begin{array}{c}-0.032 \\
(0.053)\end{array}$ & $\begin{array}{c}-0.036 \\
(0.055)\end{array}$ \\
\hline $55-64$ & $\begin{array}{c}-0.002 \\
(0.042)\end{array}$ & $\begin{array}{c}-0.004 \\
(0.042)\end{array}$ & $\begin{array}{c}-0.020 \\
(0.046)\end{array}$ & $\begin{array}{c}0.008 \\
(0.052)\end{array}$ & $\begin{array}{c}0.004 \\
(0.054)\end{array}$ \\
\hline BIP & $\begin{array}{c}0.203^{* * *} \\
(0.059)\end{array}$ & $\begin{array}{c}0.350^{* * *} \\
(0.072)\end{array}$ & $\begin{array}{c}0.326^{* * *} \\
(0.073)\end{array}$ & $\begin{array}{c}0.352^{* * *} \\
(0.077)\end{array}$ & $\begin{array}{c}0.353^{* * *} \\
(0.077)\end{array}$ \\
\hline Female & $\begin{array}{c}0.134^{* * *} \\
(0.022)\end{array}$ & $\begin{array}{c}0.148^{* * *} \\
(0.022)\end{array}$ & $\begin{array}{c}0.155^{* * *} \\
(0.023)\end{array}$ & $\begin{array}{c}0.162^{* * *} \\
(0.025)\end{array}$ & $\begin{array}{c}0.162^{* * *} \\
(0.025)\end{array}$ \\
\hline BIP $\times$ Female & & $\begin{array}{c}-0.297^{* *} \\
(0.116)\end{array}$ & $\begin{array}{c}-0.284^{* *} \\
(0.118)\end{array}$ & $\begin{array}{c}-0.267^{* *} \\
(0.125)\end{array}$ & $\begin{array}{c}-0.267^{* *} \\
(0.125)\end{array}$ \\
\hline F2F interview baseline & $\begin{array}{l}0.058^{* *} \\
(0.024)\end{array}$ & $\begin{array}{l}0.058^{* *} \\
(0.024)\end{array}$ & $\begin{array}{l}0.056^{* *} \\
(0.026)\end{array}$ & $\begin{array}{l}0.056^{* *} \\
(0.027)\end{array}$ & $\begin{array}{l}0.056^{* *} \\
(0.027)\end{array}$ \\
\hline Household size & $\begin{array}{c}0.014 \\
(0.009)\end{array}$ & $\begin{array}{c}0.014 \\
(0.009)\end{array}$ & $\begin{array}{c}0.018^{*} \\
(0.010)\end{array}$ & $\begin{array}{c}0.012 \\
(0.010)\end{array}$ & $\begin{array}{c}0.011 \\
(0.010)\end{array}$ \\
\hline Living with a partner & & & $\begin{array}{c}0.047 \\
(0.086)\end{array}$ & $\begin{array}{l}-0.015 \\
(0.091)\end{array}$ & $\begin{array}{c}-0.016 \\
(0.091)\end{array}$ \\
\hline Divorced or separated & & & $\begin{array}{c}-0.008 \\
(0.095)\end{array}$ & $\begin{array}{c}-0.036 \\
(0.099)\end{array}$ & $\begin{array}{c}-0.036 \\
(0.099)\end{array}$ \\
\hline Never married & & & $\begin{array}{c}0.111 \\
(0.094)\end{array}$ & $\begin{array}{c}0.046 \\
(0.099)\end{array}$ & $\begin{array}{c}0.046 \\
(0.099)\end{array}$ \\
\hline London & & & $\begin{array}{c}0.066 \\
(0.041)\end{array}$ & $\begin{array}{c}0.037 \\
(0.042)\end{array}$ & $\begin{array}{c}0.037 \\
(0.042)\end{array}$ \\
\hline Wales & & & $\begin{array}{c}0.085^{*} \\
(0.051)\end{array}$ & $\begin{array}{c}0.051 \\
(0.054)\end{array}$ & $\begin{array}{c}0.051 \\
(0.054)\end{array}$ \\
\hline Scotland & & & $\begin{array}{l}0.089 * * \\
(0.043)\end{array}$ & $\begin{array}{c}0.075^{*} \\
(0.045)\end{array}$ & $\begin{array}{c}0.074 \\
(0.045)\end{array}$ \\
\hline Northern Ireland & & & $\begin{array}{c}-0.037 \\
(0.065)\end{array}$ & $\begin{array}{c}0.020 \\
(0.062)\end{array}$ & $\begin{array}{c}0.020 \\
(0.062)\end{array}$ \\
\hline BA or higher & & & $\begin{array}{l}-0.010 \\
(0.037)\end{array}$ & $\begin{array}{c}-0.001 \\
(0.041)\end{array}$ & $\begin{array}{c}-0.001 \\
(0.041)\end{array}$ \\
\hline Diploma or equiv. & & & $\begin{array}{c}-0.054 \\
(0.046)\end{array}$ & $\begin{array}{c}-0.041 \\
(0.049)\end{array}$ & $\begin{array}{c}-0.041 \\
(0.049)\end{array}$ \\
\hline A Level or equiv. & & & $\begin{array}{c}-0.069 \\
(0.049)\end{array}$ & $\begin{array}{l}-0.080 \\
(0.052)\end{array}$ & $\begin{array}{c}-0.080 \\
(0.052)\end{array}$ \\
\hline GCSE or equiv. & & & $\begin{array}{l}-0.049 \\
(0.039)\end{array}$ & $\begin{array}{c}-0.052 \\
(0.040)\end{array}$ & $\begin{array}{c}-0.052 \\
(0.040)\end{array}$ \\
\hline Self-employed & & & & $\begin{array}{l}-0.008 \\
(0.057)\end{array}$ & $\begin{array}{c}-0.008 \\
(0.057)\end{array}$ \\
\hline Net personal income & & & & $\begin{array}{c}0.003 \\
(0.006)\end{array}$ & $\begin{array}{c}0.003 \\
(0.006)\end{array}$ \\
\hline Intermediate & & & & $\begin{array}{c}-0.026 \\
(0.038)\end{array}$ & $\begin{array}{l}-0.026 \\
(0.038)\end{array}$ \\
\hline
\end{tabular}


Small employers \& own account

Lower supervisory \& technical

Semi-routine \& routine

COVID-19 high risk

$$
\begin{array}{cc}
0.190^{* * *} & 0.190^{* * *} \\
(0.065) & (0.065) \\
0.058 & 0.058 \\
(0.051) & (0.051) \\
0.009 & 0.009 \\
(0.035) & (0.035) \\
& -0.008 \\
& (0.030)
\end{array}
$$

\begin{tabular}{llllll}
\hline $\mathrm{N}$ & 8,695 & 8,695 & 7,729 & 7,022 & 7,021 \\
$\mathrm{R}$-squared & 0.015 & 0.016 & 0.020 & 0.022 & 0.022 \\
\hline
\end{tabular}


Table A5: OLS regressions of percentage point changes in mental health problems in the UKHLS from Wave 9 (2017-2019) to April 2020 on White and other characteristics

\begin{tabular}{|c|c|c|c|c|c|}
\hline & (1) & $(2)$ & (3) & (4) & (5) \\
\hline$<25$ & $\begin{array}{c}0.016 \\
(0.023)\end{array}$ & $\begin{array}{c}0.017 \\
(0.023)\end{array}$ & $\begin{array}{c}0.004 \\
(0.030)\end{array}$ & $\begin{array}{c}0.018 \\
(0.040)\end{array}$ & $\begin{array}{c}0.021 \\
(0.041)\end{array}$ \\
\hline $25-34$ & $\begin{array}{c}0.060^{* * *} \\
(0.018)\end{array}$ & $\begin{array}{c}0.061^{* * *} \\
(0.018)\end{array}$ & $\begin{array}{l}0.042^{* *} \\
(0.020)\end{array}$ & $\begin{array}{c}0.074^{* * *} \\
(0.026)\end{array}$ & $\begin{array}{c}0.077^{* * *} \\
(0.027)\end{array}$ \\
\hline $35-44$ & $\begin{array}{c}0.020 \\
(0.016)\end{array}$ & $\begin{array}{c}0.020 \\
(0.016)\end{array}$ & $\begin{array}{c}0.010 \\
(0.018)\end{array}$ & $\begin{array}{c}0.042^{*} \\
(0.024)\end{array}$ & $\begin{array}{c}0.045^{*} \\
(0.025)\end{array}$ \\
\hline $45-54$ & $\begin{array}{c}-0.010 \\
(0.014)\end{array}$ & $\begin{array}{l}-0.011 \\
(0.014)\end{array}$ & $\begin{array}{c}-0.016 \\
(0.016)\end{array}$ & $\begin{array}{c}0.016 \\
(0.022)\end{array}$ & $\begin{array}{c}0.019 \\
(0.023)\end{array}$ \\
\hline $55-64$ & $\begin{array}{c}-0.017 \\
(0.013)\end{array}$ & $\begin{array}{l}-0.017 \\
(0.013)\end{array}$ & $\begin{array}{c}-0.015 \\
(0.014)\end{array}$ & $\begin{array}{c}0.010 \\
(0.019)\end{array}$ & $\begin{array}{c}0.012 \\
(0.019)\end{array}$ \\
\hline White & $\begin{array}{c}0.012 \\
(0.016)\end{array}$ & $\begin{array}{c}-0.049^{* *} \\
(0.023)\end{array}$ & $\begin{array}{c}-0.031 \\
(0.025)\end{array}$ & $\begin{array}{l}-0.044^{*} \\
(0.026)\end{array}$ & $\begin{array}{l}-0.044^{*} \\
(0.026)\end{array}$ \\
\hline Female & $\begin{array}{c}0.075^{* * *} \\
(0.009)\end{array}$ & $\begin{array}{c}-0.016 \\
(0.030)\end{array}$ & $\begin{array}{c}-0.016 \\
(0.031)\end{array}$ & $\begin{array}{c}-0.020 \\
(0.032)\end{array}$ & $\begin{array}{c}-0.020 \\
(0.032)\end{array}$ \\
\hline White $\times$ Female & & $\begin{array}{c}0.101^{* * *} \\
(0.031)\end{array}$ & $\begin{array}{c}0.102^{* * *} \\
(0.032)\end{array}$ & $\begin{array}{c}0.108^{* * *} \\
(0.034)\end{array}$ & $\begin{array}{c}0.108^{* * *} \\
(0.034)\end{array}$ \\
\hline F2F interview baseline & $\begin{array}{c}0.005 \\
(0.010)\end{array}$ & $\begin{array}{c}0.005 \\
(0.010)\end{array}$ & $\begin{array}{c}0.010 \\
(0.011)\end{array}$ & $\begin{array}{c}0.011 \\
(0.011)\end{array}$ & $\begin{array}{c}0.011 \\
(0.011)\end{array}$ \\
\hline Household size & $\begin{array}{c}0.005 \\
(0.004)\end{array}$ & $\begin{array}{c}0.005 \\
(0.004)\end{array}$ & $\begin{array}{c}0.005 \\
(0.005)\end{array}$ & $\begin{array}{c}0.004 \\
(0.005)\end{array}$ & $\begin{array}{c}0.004 \\
(0.005)\end{array}$ \\
\hline Living with a partner & & & $\begin{array}{c}-0.003 \\
(0.029)\end{array}$ & $\begin{array}{l}-0.009 \\
(0.029)\end{array}$ & $\begin{array}{c}-0.009 \\
(0.029)\end{array}$ \\
\hline Divorced or separated & & & $\begin{array}{c}-0.011 \\
(0.033)\end{array}$ & $\begin{array}{l}-0.006 \\
(0.034)\end{array}$ & $\begin{array}{l}-0.005 \\
(0.034)\end{array}$ \\
\hline Never married & & & $\begin{array}{c}0.010 \\
(0.033)\end{array}$ & $\begin{array}{c}0.014 \\
(0.034)\end{array}$ & $\begin{array}{c}0.014 \\
(0.034)\end{array}$ \\
\hline London & & & $\begin{array}{l}0.047^{* *} \\
(0.018)\end{array}$ & $\begin{array}{l}0.046^{* *} \\
(0.019)\end{array}$ & $\begin{array}{l}0.046^{* *} \\
(0.019)\end{array}$ \\
\hline Wales & & & $\begin{array}{c}0.004 \\
(0.024)\end{array}$ & $\begin{array}{c}0.008 \\
(0.024)\end{array}$ & $\begin{array}{c}0.008 \\
(0.024)\end{array}$ \\
\hline Scotland & & & $\begin{array}{c}0.033^{*} \\
(0.019)\end{array}$ & $\begin{array}{c}0.029 \\
(0.019)\end{array}$ & $\begin{array}{c}0.029 \\
(0.019)\end{array}$ \\
\hline Northern Ireland & & & $\begin{array}{c}0.010 \\
(0.028)\end{array}$ & $\begin{array}{c}0.008 \\
(0.028)\end{array}$ & $\begin{array}{c}0.008 \\
(0.028)\end{array}$ \\
\hline BA or higher & & & $\begin{array}{c}0.063^{* * *} \\
(0.015)\end{array}$ & $\begin{array}{c}0.051^{* * *} \\
(0.015)\end{array}$ & $\begin{array}{c}0.051^{* * *} \\
(0.015)\end{array}$ \\
\hline Diploma or equiv. & & & $\begin{array}{c}0.034^{*} \\
(0.018)\end{array}$ & $\begin{array}{c}0.027 \\
(0.019)\end{array}$ & $\begin{array}{c}0.028 \\
(0.019)\end{array}$ \\
\hline A Level or equiv. & & & $\begin{array}{c}0.037^{*} \\
(0.019)\end{array}$ & $\begin{array}{c}0.028 \\
(0.020)\end{array}$ & $\begin{array}{c}0.028 \\
(0.020)\end{array}$ \\
\hline GCSE or equiv. & & & $\begin{array}{c}0.011 \\
(0.015)\end{array}$ & $\begin{array}{c}0.007 \\
(0.015)\end{array}$ & $\begin{array}{c}0.007 \\
(0.015)\end{array}$ \\
\hline Self-employed & & & & $\begin{array}{c}0.024 \\
(0.019)\end{array}$ & $\begin{array}{c}0.024 \\
(0.019)\end{array}$ \\
\hline Unemployed & & & & $\begin{array}{c}-0.121^{* * *} \\
(0.033)\end{array}$ & $\begin{array}{c}-0.121^{* * *} \\
(0.033)\end{array}$ \\
\hline Retired & & & & $\begin{array}{c}0.032^{*} \\
(0.019)\end{array}$ & $\begin{array}{c}0.031 \\
(0.019)\end{array}$ \\
\hline House-keeper & & & & 0.007 & 0.007 \\
\hline
\end{tabular}


Student

Disabled

Other

Net personal income

COVID-19 high risk

$\begin{array}{cc}(0.029) & (0.029) \\ 0.032 & 0.032 \\ (0.039) & (0.039) \\ -0.159 * * * & -0.161^{* * *} \\ (0.037) & (0.037) \\ 0.062 & 0.062 \\ (0.062) & (0.062) \\ 0.002 & 0.002 \\ (0.003) & (0.003) \\ & 0.005 \\ & (0.013)\end{array}$

14,155

14,155

12,380

12,000

0.011

0.015

0.008

0.009

11,999
0.015

0.011


Table A6: OLS regressions of percentage point changes in mental health problems in the UKHLS from Wave 9 (2017-2019) to April 2020 on BIP and other characteristics

\begin{tabular}{|c|c|c|c|c|c|}
\hline & $(1)$ & $(2)$ & (3) & (4) & $(5)$ \\
\hline \multirow[t]{2}{*}{$<25$} & 0.013 & 0.014 & 0.003 & 0.017 & 0.019 \\
\hline & $(0.023)$ & $(0.023)$ & $(0.030)$ & $(0.040)$ & $(0.041)$ \\
\hline \multirow[t]{2}{*}{$25-34$} & $0.058^{* * *}$ & $0.059 * * *$ & $0.040^{* *}$ & $0.072^{* * *}$ & $0.074^{* * *}$ \\
\hline & $(0.018)$ & $(0.018)$ & $(0.020)$ & $(0.026)$ & $(0.027)$ \\
\hline \multirow[t]{2}{*}{$35-44$} & 0.019 & 0.018 & 0.008 & $0.040^{*}$ & $0.043^{*}$ \\
\hline & $(0.016)$ & $(0.016)$ & $(0.018)$ & $(0.024)$ & $(0.025)$ \\
\hline \multirow[t]{2}{*}{$45-54$} & -0.011 & -0.011 & -0.017 & 0.015 & 0.017 \\
\hline & $(0.014)$ & $(0.014)$ & $(0.016)$ & $(0.022)$ & $(0.023)$ \\
\hline \multirow[t]{2}{*}{$55-64$} & -0.017 & -0.017 & -0.016 & 0.009 & 0.011 \\
\hline & $(0.013)$ & $(0.013)$ & $(0.014)$ & $(0.019)$ & $(0.019)$ \\
\hline \multirow[t]{2}{*}{ BIP } & 0.027 & $0.104^{* * *}$ & $0.090^{* * *}$ & $0.104^{* * *}$ & $0.104^{* * *}$ \\
\hline & $(0.022)$ & $(0.030)$ & $(0.032)$ & $(0.033)$ & $(0.033)$ \\
\hline \multirow[t]{2}{*}{ Female } & $0.075^{* * *}$ & $0.082^{* * *}$ & $0.083^{* * *}$ & $0.085^{* * *}$ & $0.085^{* * *}$ \\
\hline & $(0.009)$ & $(0.009)$ & $(0.010)$ & $(0.011)$ & $(0.011)$ \\
\hline \multirow[t]{2}{*}{ BIP $\times$ Female } & & $-0.135^{* * *}$ & $-0.132 * * *$ & $-0.135^{* * *}$ & $-0.135^{* * *}$ \\
\hline & & $(0.041)$ & $(0.043)$ & $(0.045)$ & $(0.045)$ \\
\hline \multirow[t]{2}{*}{ F2F interview baseline } & 0.004 & 0.004 & 0.009 & 0.010 & 0.011 \\
\hline & $(0.010)$ & $(0.010)$ & $(0.011)$ & $(0.011)$ & $(0.011)$ \\
\hline \multirow[t]{2}{*}{ Household size } & 0.004 & 0.004 & 0.003 & 0.003 & 0.003 \\
\hline & $(0.004)$ & $(0.004)$ & $(0.005)$ & $(0.005)$ & $(0.005)$ \\
\hline \multirow[t]{2}{*}{ Living with a partner } & & & -0.002 & -0.009 & -0.008 \\
\hline & & & $(0.029)$ & $(0.029)$ & $(0.029)$ \\
\hline \multirow[t]{2}{*}{ Divorced or separated } & & & -0.013 & -0.007 & -0.007 \\
\hline & & & $(0.033)$ & $(0.034)$ & $(0.034)$ \\
\hline \multirow[t]{2}{*}{ Never married } & & & 0.007 & 0.012 & 0.013 \\
\hline & & & $(0.033)$ & $(0.034)$ & $(0.034)$ \\
\hline \multirow[t]{2}{*}{ London } & & & $0.035^{* *}$ & $0.036^{* *}$ & $0.036^{* *}$ \\
\hline & & & $(0.018)$ & $(0.018)$ & $(0.018)$ \\
\hline \multirow[t]{2}{*}{ Wales } & & & 0.006 & 0.011 & 0.011 \\
\hline & & & $(0.024)$ & $(0.024)$ & $(0.024)$ \\
\hline \multirow[t]{2}{*}{ Scotland } & & & $0.036^{*}$ & $0.031^{*}$ & $0.031^{*}$ \\
\hline & & & $(0.019)$ & $(0.019)$ & $(0.019)$ \\
\hline \multirow[t]{2}{*}{ Northern Ireland } & & & 0.014 & 0.011 & 0.011 \\
\hline & & & $(0.028)$ & $(0.028)$ & $(0.028)$ \\
\hline \multirow[t]{2}{*}{ BA or higher } & & & $0.062^{* * *}$ & $0.049 * * *$ & $0.050 * * *$ \\
\hline & & & $(0.015)$ & $(0.015)$ & $(0.015)$ \\
\hline \multirow[t]{2}{*}{ Diploma or equiv. } & & & $0.033^{*}$ & 0.027 & 0.027 \\
\hline & & & $(0.018)$ & $(0.019)$ & $(0.019)$ \\
\hline \multirow[t]{2}{*}{ A Level or equiv. } & & & $0.036^{*}$ & 0.026 & 0.027 \\
\hline & & & $(0.019)$ & $(0.020)$ & $(0.020)$ \\
\hline \multirow[t]{2}{*}{ GCSE or equiv. } & & & 0.011 & 0.007 & 0.007 \\
\hline & & & $(0.015)$ & $(0.015)$ & $(0.015)$ \\
\hline Self-employed & & & & 0.023 & 0.023 \\
\hline & & & & $(0.019)$ & $(0.019)$ \\
\hline Unemployed & & & & $-0.122 * * *$ & $-0.122^{* * *}$ \\
\hline & & & & $(0.033)$ & $(0.033)$ \\
\hline Retired & & & & 0.031 & 0.030 \\
\hline & & & & $(0.019)$ & $(0.019)$ \\
\hline House-keeper & & & & 0.004 & 0.004 \\
\hline
\end{tabular}


Student

Disabled

Other

Net personal income

COVID-19 high risk

$\begin{array}{cc}(0.029) & (0.029) \\ 0.030 & 0.030 \\ (0.039) & (0.039) \\ -0.162^{* * *} & -0.163^{* * *} \\ (0.037) & (0.037) \\ 0.062 & 0.062 \\ (0.062) & (0.062) \\ 0.002 & 0.002 \\ (0.003) & (0.003) \\ & 0.004 \\ & (0.013)\end{array}$

$\mathrm{N}$

14,155

14,155

0.009

12,380

12,000

11,999

R-squared

0.008

0.011

0.015

0.015 
Table A7: OLS regressions of percentage point changes in mental health problems from Wave 9 (2017-2019) to April 2020 on White and other characteristics

(1)

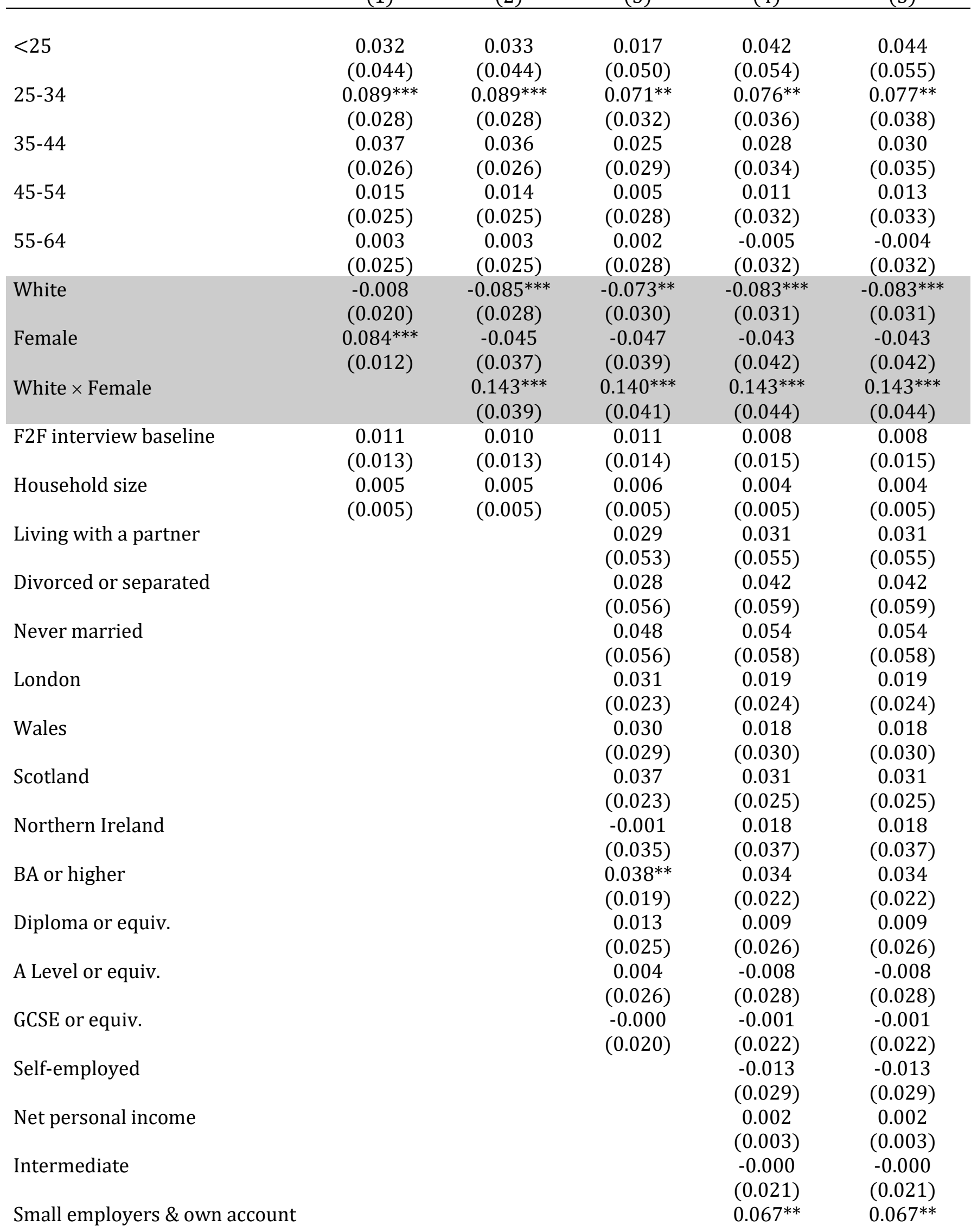


Lower supervisory \& technical

$(0.034) \quad(0.034)$

$0.020 \quad 0.020$

$(0.029) \quad(0.029)$

Semi-routine \& routine

$-0.014 \quad-0.014$

(0.019) (0.019)

COVID-19 high risk

0.002

N

R-squared

8,695

8,695

7,729

7,022

0.013

0.014

7,021

0.010

0.012

0.014 
Table A8: OLS regressions of percentage point changes in mental health problems from Wave 9 (2017-2019) to April 2020 on BIP and other characteristics

\begin{tabular}{|c|c|c|c|c|c|}
\hline & (1) & (2) & (3) & (4) & (5) \\
\hline \multirow[t]{2}{*}{$<25$} & 0.029 & 0.030 & 0.015 & 0.038 & 0.039 \\
\hline & $(0.044)$ & $(0.044)$ & $(0.050)$ & $(0.054)$ & $(0.055)$ \\
\hline \multirow[t]{2}{*}{$25-34$} & $0.087^{* * *}$ & $0.087^{* * *}$ & $0.070^{* *}$ & $0.073^{* *}$ & $0.074^{* *}$ \\
\hline & $(0.028)$ & $(0.028)$ & $(0.032)$ & $(0.036)$ & $(0.038)$ \\
\hline \multirow[t]{2}{*}{$35-44$} & 0.036 & 0.034 & 0.023 & 0.026 & 0.027 \\
\hline & $(0.026)$ & $(0.026)$ & $(0.029)$ & $(0.034)$ & $(0.035)$ \\
\hline \multirow[t]{2}{*}{$45-54$} & 0.015 & 0.013 & 0.004 & 0.010 & 0.011 \\
\hline & $(0.025)$ & $(0.025)$ & $(0.028)$ & $(0.032)$ & $(0.033)$ \\
\hline \multirow[t]{2}{*}{$55-64$} & 0.004 & 0.002 & 0.001 & -0.006 & -0.006 \\
\hline & $(0.025)$ & $(0.025)$ & $(0.028)$ & $(0.032)$ & $(0.032)$ \\
\hline \multirow[t]{2}{*}{ BIP } & $0.081^{* * *}$ & $0.167^{* * *}$ & $0.158^{* * *}$ & $0.175^{* * *}$ & $0.175^{* * *}$ \\
\hline & $(0.028)$ & $(0.036)$ & $(0.037)$ & $(0.040)$ & $(0.040)$ \\
\hline \multirow[t]{2}{*}{ Female } & $0.085^{* * *}$ & $0.093^{* * *}$ & $0.088^{* * *}$ & $0.095^{* * *}$ & $0.095^{* * *}$ \\
\hline & $(0.012)$ & $(0.012)$ & $(0.013)$ & $(0.014)$ & $(0.014)$ \\
\hline \multirow[t]{2}{*}{ BIP $\times$ Female } & & $-0.173^{* * *}$ & $-0.167^{* * *}$ & $-0.169 * * *$ & $-0.170^{* * *}$ \\
\hline & & $(0.055)$ & $(0.056)$ & $(0.061)$ & $(0.061)$ \\
\hline \multirow[t]{2}{*}{ F2F interview baseline } & 0.010 & 0.010 & 0.010 & 0.007 & 0.007 \\
\hline & $(0.013)$ & $(0.013)$ & $(0.014)$ & $(0.015)$ & $(0.015)$ \\
\hline \multirow[t]{2}{*}{ Household size } & 0.003 & 0.003 & 0.005 & 0.002 & 0.002 \\
\hline & $(0.005)$ & $(0.005)$ & $(0.005)$ & $(0.005)$ & $(0.005)$ \\
\hline \multirow[t]{2}{*}{ Living with a partner } & & & 0.030 & 0.032 & 0.032 \\
\hline & & & $(0.053)$ & $(0.055)$ & $(0.055)$ \\
\hline \multirow[t]{2}{*}{ Divorced or separated } & & & 0.026 & 0.040 & 0.040 \\
\hline & & & $(0.057)$ & $(0.059)$ & $(0.059)$ \\
\hline \multirow[t]{2}{*}{ Never married } & & & 0.047 & 0.054 & 0.054 \\
\hline & & & $(0.056)$ & $(0.058)$ & $(0.058)$ \\
\hline \multirow[t]{2}{*}{ London } & & & 0.021 & 0.010 & 0.010 \\
\hline & & & $(0.022)$ & $(0.023)$ & $(0.023)$ \\
\hline \multirow[t]{2}{*}{ Wales } & & & 0.033 & 0.021 & 0.021 \\
\hline & & & $(0.029)$ & $(0.031)$ & $(0.031)$ \\
\hline \multirow[t]{2}{*}{ Scotland } & & & $0.041^{*}$ & 0.035 & 0.035 \\
\hline & & & $(0.023)$ & $(0.025)$ & $(0.025)$ \\
\hline \multirow[t]{2}{*}{ Northern Ireland } & & & 0.003 & 0.022 & 0.022 \\
\hline & & & $(0.035)$ & $(0.037)$ & $(0.037)$ \\
\hline \multirow[t]{2}{*}{ BA or higher } & & & $0.037 *$ & 0.033 & 0.033 \\
\hline & & & $(0.019)$ & $(0.022)$ & $(0.022)$ \\
\hline \multirow[t]{2}{*}{ Diploma or equiv. } & & & 0.012 & 0.008 & 0.008 \\
\hline & & & $(0.025)$ & $(0.026)$ & $(0.026)$ \\
\hline \multirow[t]{2}{*}{ A Level or equiv. } & & & 0.001 & -0.010 & -0.010 \\
\hline & & & $(0.026)$ & $(0.028)$ & $(0.028)$ \\
\hline \multirow[t]{2}{*}{ GCSE or equiv. } & & & 0.000 & -0.001 & -0.001 \\
\hline & & & $(0.020)$ & $(0.022)$ & $(0.022)$ \\
\hline Self-employed & & & & -0.015 & -0.015 \\
\hline & & & & $(0.029)$ & $(0.029)$ \\
\hline Net personal income & & & & 0.002 & 0.002 \\
\hline & & & & $(0.003)$ & $(0.003)$ \\
\hline Intermediate & & & & -0.002 & -0.002 \\
\hline & & & & $(0.021)$ & $(0.021)$ \\
\hline Small employers \& own account & & & & $0.068^{* *}$ & $0.068^{* *}$ \\
\hline
\end{tabular}


Lower supervisory \& technical

Semi-routine \& routine

COVID-19 high risk
$(0.034) \quad(0.034)$

$0.020 \quad 0.020$

(0.029) (0.029)

$-0.015 \quad-0.015$

(0.019) (0.019)

0.001

(0.016)

\begin{tabular}{llllll}
\hline $\mathrm{N}$ & 8,695 & 8,695 & 7,729 & 7,022 & 7,021 \\
$\mathrm{R}$-squared & 0.011 & 0.012 & 0.013 & 0.015 & 0.015 \\
\hline
\end{tabular}

